\title{
RNA-binding activity of TRIM25 is mediated by its PRY/SPRY domain and is required for ubiquitination
}

\author{
Nila Roy Choudhury', Gregory Heikel', Maryia Trubitsyna², Peter Kubik', Jakub Stanislaw Nowak', Shaun Webb, \\ Sander Granneman ${ }^{3}$, Christos Spanos ${ }^{1}$, Juri Rappsilber ${ }^{1,4}$, Alfredo Castello ${ }^{5}$ and Gracjan Michlewski ${ }^{1 *}$ (D)
}

\begin{abstract}
Background: TRIM25 is a novel RNA-binding protein and a member of the Tripartite Motif (TRIM) family of E3 ubiquitin ligases, which plays a pivotal role in the innate immune response. However, there is scarce knowledge about its RNArelated roles in cell biology. Furthermore, its RNA-binding domain has not been characterized.

Results: Here, we reveal that the RNA-binding activity of TRIM25 is mediated by its PRY/SPRY domain, which we postulate to be a novel RNA-binding domain. Using CLIP-seq and SILAC-based co-immunoprecipitation assays, we uncover TRIM25's endogenous RNA targets and protein binding partners. We demonstrate that TRIM25 controls the levels of Zinc Finger Antiviral Protein (ZAP). Finally, we show that the RNA-binding activity of TRIM25 is important for its ubiquitin ligase activity towards itself (autoubiquitination) and its physiologically relevant target ZAP.

Conclusions: Our results suggest that many other proteins with the PRY/SPRY domain could have yet uncharacterized RNA-binding potential. Together, our data reveal new insights into the molecular roles and characteristics of RNA-binding E3 ubiquitin ligases and demonstrate that RNA could be an essential factor in their enzymatic activity.
\end{abstract}

Keywords: Novel RNA-binding proteins, TRIM25, ZAP, RNA-binding domain, PRY/SPRY

\section{Background}

More than 300 novel RNA-binding proteins have been identified in HeLa [1], HEK293 [2], and mouse ES cells [3]. Furthermore, a recent study identified 393 new RNA-binding proteins unique to cardiomyocytes [4]. Strikingly, many of these proteins were not previously known for their RNA-binding properties and do not contain canonical RNA-binding domains. Intriguingly, E3 ubiquitin ligases from the Tripartite Motif (TRIM) family (TRIM25, TRIM56, and TRIM71) are among these newly identified RNA-binding proteins. TRIM proteins have a characteristic architecture that includes RING zinc-finger, B-Box, coiled-coil (CC), and variable C-terminal domains. There are over 80 TRIM proteins in the human genome, and they have been divided into 11 subfamilies based on their structural composition [5].

\footnotetext{
* Correspondence: gracjan.michlewski@ed.ac.uk

${ }^{1}$ Wellcome Centre for Cell Biology, University of Edinburgh, Michael Swann

Building, Edinburgh EH9 3BF, UK

Full list of author information is available at the end of the article
}

The largest subfamily (C-IV) of 33 TRIM proteins is characterized by the PRY/SPRY domain at the Cterminal end. Most proteins from the TRIM family are involved in innate immune responses [6, 7]. For example, TRIM25 plays a role in the Retinoic AcidInducible Gene 1 (RIG-I)-mediated interferon response to viral RNAs $[5,8]$. RIG-I recognizes viral RNA molecules with a $5^{\prime}$-triphosphate (5'-ppp-RNA) [9] and initiates a downstream signaling cascade that culminates with the expression of type I interferons (interferon $\alpha$ and $\beta$ ) [10] and an anti-viral response. TRIM25 mediates K63-linked ubiquitination of RIG-I, which leads to efficient recruitment of its downstream partners. Such recruitment in turn triggers the interferon response [11]. Recently, TRIM25 was shown to be required for the antiviral activity of RNA-binding Zinc Finger Antiviral Protein (ZAP) [12, 13]. However, little is known about the RNA-mediated roles and characteristics of these RNA-binding E3 ligases. 
Although TRIM25 has been generally well characterized, the functional importance of its RNA-binding activity remains unclear. Our recent study provided the first clues, showing that the binding of TRIM25 to pre-let-7 leads to its more efficient Lin28a-mediated uridylation [14]. This finding raised the possibility that RNA processing may be spatially controlled through post-translational protein modifications by RNA-binding protein-modifying enzymes such as TRIM25. Furthermore, TRIM25 could be using RNA as a scaffold to ubiquitinate its targets. Importantly, the transcriptome and proteome-wide targets of TRIM25 have not yet been identified. Finally, its RNA-binding domain has not been unequivocally described.

Herein, using data from a proteome-wide analysis of RNA-binding domains, RNA pull-downs, and an electrophoretic mobility shift assay (EMSA), we show that the PRY/SPRY domain of TRIM25 harbors its RNA-binding activity. Furthermore, TRIM25 CLIP-seq and quantitative SILAC-based co-immunoprecipitation assays reveal a comprehensive overview of interacting RNAs and proteins, respectively. Our CLIP-seq data identifies hundreds of coding and non-coding RNAs bound by TRIM25. We show that TRIM25 binds a distinct set of RNAs and prefers to bind GC-rich sequences. We also demonstrate that TRIM25 associates with many RNA-binding proteins, thus suggesting it could play a role in regulating RNA metabolism. Crucially, we present evidence that the binding of TRIM25 to RNA is important for its ubiquitin ligase activity towards itself (autoubiquitination) and its physiologically relevant target ZAP. Finally, by showing that the PRY/ SPRY regions of other TRIM proteins rescue TRIM25's RNA-binding activity, we propose that TRIM25 represents a novel group of E3 ligases with RNA-binding activity that use PRY/SPRY as a novel RNA-binding domain. These results provide original insights into the RNA-mediated activities of RNA-binding E3 ubiquitin ligases.

\section{Results}

TRIM25 directly interacts with RNA through the PRY/SPRY domain

TRIM25 is known to associate with RNAs in cells and cell extracts $[3,14]$. However, whether this interaction is direct or indirect remains unknown. To test this, we performed an EMSA with pre-let-7a-1 and purified recombinant human His-tagged TRIM25, expressed in E. coli (Additional file 1: Figure S1a). EMSA, with increasing amounts of His-TRIM25, revealed that TRIM25 interacts with RNA directly (Fig. 1a), with an observed $\mathrm{Kd}$ of $800 \mathrm{nM}$. This showed that TRIM25 was a bona fide RNA-binding protein.

TRIM25 has been previously shown to bind RNA through its coiled-coil CC domain [3], which is responsible for its dimerization and oligomerization [15-18]. Surprisingly, a proteome-wide analysis of RNA-binding domains (RBDs) in HeLa cells revealed that the TRIM25 PRY/SPRY domain may be responsible for direct binding to RNA (Fig. 1b) [19]. In brief, this method combines UV protein-RNA crosslinking, oligo $(\mathrm{dT})$ capture, and controlled proteolysis for a high-resolution ( 17 amino acids on average) delineation of the protein region engaged in RNA binding, which in the case of TRIM25, is the peptide enriched in the RNA-bound fraction mapped to the PRY/SPRY domain (TRIM25 residues 470-508), demonstrating direct RNA binding (red line; $1 \%$ false discovery rate, Fig. 1b). In contrast, peptides mapping to other regions of the protein, including the CC domain, were released into the supernatant following protease cleavage (blue lines), suggesting a lack of RNAbinding activity (Fig. 1b). To validate this, we expressed and purified a recombinant His-TRIM25 deletion mutant (lacking residues 470-508 located in the PRY/SPRY domain) (Additional file 1: Figure S1a), which we tentatively named TRIM25 $\triangle$ RBD, and tested its binding activity in vitro (Fig. 1c, d). EMSA analysis with increasing amounts of His-TRIM25 $\triangle$ RBD revealed no shift in the pre-let-7a-1 substrate (Fig. 1c). Likewise, RNA pulldown assays in HeLa cell extracts showed that endogenous and T7-tagged TRIM25 were efficiently pulled-down with pre-let-7a-1, whereas T7-tagged TRIM $25 \Delta$ RBD was not (Fig. 1e). These results strongly indicate that the TRIM25 PRY/SPRY domain harbors its RNA-binding activity.

Importantly, some RNA-binding proteins must dimerize to be able to bind RNA [20, 21]. Since TRIM25 is known to dimerize and tetramerize through its CC domain with minimal contributions from the PRY/SPRY [15-18], we decided to generate TRIM25 knockout (KO) cells, which removed any confounding effects of endogenous TRIM25. To obtain TRIM25 KO cells, we used CRISPR/Cas9 targeting followed by clonal selection and dot blot analysis. This strategy yielded several HeLa cell clones with complete loss of TRIM25 expression (Additional file 1: Figure S2a). For further analysis, we selected clone C3 (Additional file 1: Figure S2b). Next, we performed RNA pull-down assays in extracts from the TRIM25 KO cells with T7TRIM25 $\triangle$ RBD and additional TRIM25 truncations (Fig. 1d). These experiments confirmed that neither T7-TRIM25 $\triangle$ RBD nor T7-TRIM25 $\Delta$ CC retained RNAbinding activity (Fig. 1f). Importantly, neither T7TRIM25 $\triangle$ PRY/SPRY nor T7-TRIM25-PRY/SPRY could bind RNA (Fig. 1g), indicating that the TRIM25 CC domain is essential, whereas the isolated PRY/SPRY domain is not sufficient for binding to RNA. These results suggest that TRIM25 binding to RNA could be dependent on its CC-mediated dimerization and are in line with previous results, which linked the CC domain with TRIM25's RNA-binding activity [3]. 
a

His-TRIM25

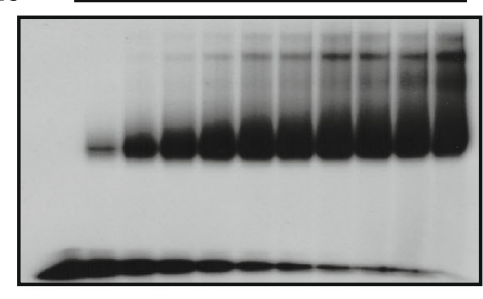

$\begin{array}{lllllllllll}1 & 2 & 3 & 4 & 5 & 6 & 7 & 8 & 9 & 10 & 11\end{array}$

b
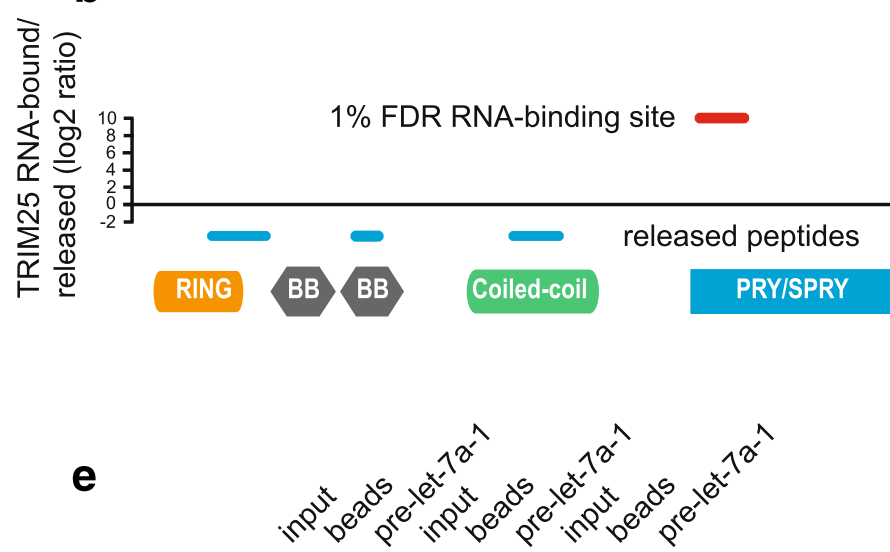

T7-TRIM25 WT - - $+++-\quad-$

T7-TRIM25 $\triangle$ RBD - $\quad-\quad-\quad-\quad+++$

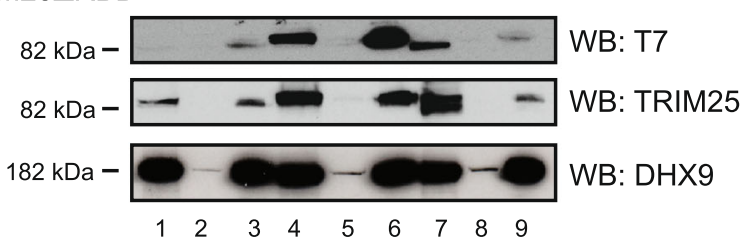

pre-let-7a-1/

TRIM25

pre-let-7a-1
C

His-TRIM25 $\triangle$ RBD

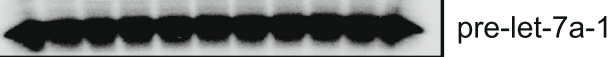

$\begin{array}{lllllllllll}1 & 2 & 3 & 4 & 5 & 6 & 7 & 8 & 9 & 10 & 11\end{array}$

d
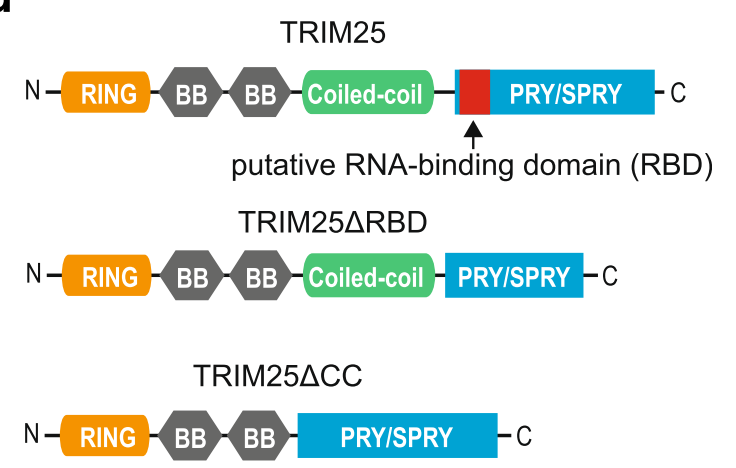

TRIM25 $\triangle \mathrm{PRY} / \mathrm{SPRY}$

$\mathrm{N}-$ RING - BB - BB -Coiled-coil - C

TRIM25-PRY/SPRY

N- PRY/SPRY - C

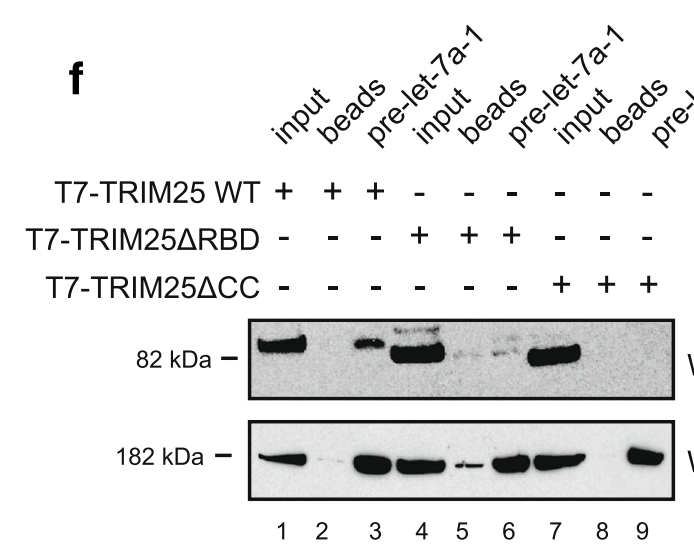

g

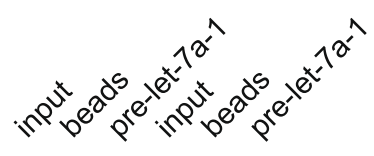

T7-TRIM25 $\triangle \mathrm{PRY} / \mathrm{SPRY}+++$ + -

T7-TRIM25-PRY/SPRY - - - + + +

WB: T7

WB: DHX9

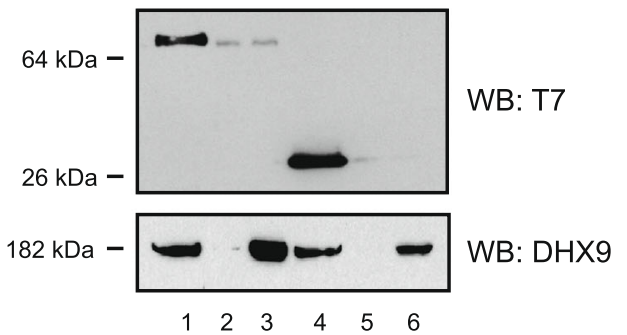

Fig. 1 (See legend on next page.) 


\begin{abstract}
(See figure on previous page.)
Fig. 1 TRIM25 is a bona fide RNA-binding protein and the PRY/SPRY domain is responsible for binding to RNA. a EMSA analysis of recombinant His-tagged TRIM25 with pre-let-7a-1. Lane 1 represents the loading control. Lanes 2-11 show EMSA with increasing amounts of TRIM25 (200, 400 , $600,800,1000,1200,1400,1600,1800$, and 2000 ng). b RNA capture assay result for TRIM25. Red line represents peptide enriched in the RNAbound fraction. Blue lines show peptides depleted from the RNA-bound fraction. c EMSA analysis of recombinant His-tagged TRIM25 RRBD with pre-let-7a-1. Lane 1 represents the loading control. Lanes 2-11 show EMSA with increasing amounts of TRIM25 $\Delta$ RBD $(200,400,600,800,1000$, 1200, 1400, 1600, 1800, and 2000 ng). d Domain architecture of wild-type TRIM25 and deletion mutants. Relative position of RNA-enriched peptide is shown in red. e Western blot analyses against T7, TRIM25, and DHX9 of pre-let-7a-1 pull-downs with HeLa cell extracts overexpressing T7-TRIM25 or T7TRIM25 $\triangle$ RBD. Lanes 1, 4, and 7 represent 4\% (40 $\mu \mathrm{g}$ ) of the loading controls (input). Lanes 2, 5, and 8 represent bead-only pull-downs. Lanes 3, 6, and 9 show pre-let-7a-1 pull-down. Note that T7 antibody highlights an unspecific band visible in lanes 3 and 8. $\mathbf{f}$ Western blot analyses against T7 and DHX9 of pre-let-7a-1 pull-downs with HeLa TRIM25 KO cell extracts overexpressing T7-TRIM25, T7-TRIM25 $\Delta$ RBD, or T7-TRIM25 $\Delta$ CC. Lanes 1, 4, and 7 represent $4 \%(40 \mu \mathrm{g})$ of the loading controls (input). Lanes 2, 5, and 8 represent bead-only pull-downs. Lanes 3, 6, and 9 show pre-let-7a- 1 pull-down. $\mathbf{g ~ W e s t e r n ~}$ blot analyses against T7 and DHX9 of pre-let-7a-1 pull-downs with HeLa TRIM25 KO cell extracts overexpressing T7-TRIM25 PPRY/SPRY or T7-TRIM25PRY/SPR. Lanes 1 and 4 represent $4 \%(40 \mu \mathrm{g})$ of the loading controls (input). Lanes 2 and 5 represent bead-only controls. Lanes 3 and 6 show pre-let-7a-1 pull-down
\end{abstract}

Finally, we wanted to check if TRIM25 $\triangle$ RBD could fold correctly and form multimeric complexes. To test this, we overexpressed T7-tagged TRIM25 with EGFP-tagged TRIM25 (with or without the $\triangle$ RBD mutation) in TRIM25 $\mathrm{KO}$ cells and performed anti-T7 co-immunoprecipitation (co-IP) assays (Fig. 2a). Our results showed that both wildtype T7-TRIM25 and T7-TRIM25 $\triangle$ RBD could interact with EGFP-TRIM25 (Fig. 2a). The control experiment revealed that the T7 antibody led to co-IP of T7-tagged but not EGFP-tagged TRIM25 (Fig. 2b). Moreover, we confirmed that T7-TRIM25 $\triangle C C$ or the isolated T7-TRIM25PRY/SPRY domain could not form multimeric complexes with EGFP-TRIM25 (Fig. 2c). Importantly, both the wildtype and TRIM25 $\triangle$ RBD recombinant proteins displayed a similar melting temperature $\left(43^{\circ} \mathrm{C}\right)$ in a thermal denaturation assay (Additional file 1: Figure S1b). Furthermore, using size exclusion chromatography with multi-angle light scattering (MALS), we established that there was no difference in the elution profiles between the wild-type and the mutant protein (Additional file 1: Figure S1c). The MALSmeasured mass distribution for His-TRIM25 was $263 \pm$ $5.3 \mathrm{kDa}$, with an $\mathrm{Mw} / \mathrm{Mn}$ of approximately 1.01 . The HisTRIM25 $\triangle$ RBD had values of $258 \pm 5 \mathrm{kDa}$, and $\geq 1.003 \mathrm{Mw} /$ $\mathrm{Mn}$. This suggests that both wild-type and TRIM25 $\triangle$ RBD formed tetramers in our preparation. Taken together, these results show that TRIM25 harboring a $\triangle$ RBD mutation still supports interactions in trans with another TRIM25 molecule and that the $\triangle \mathrm{RBD}$ mutation does not influence the overall folding or multimerization of the TRIM 25 protein.

\section{TRIM25 co-immunoprecipitates with proteins involved in RNA metabolism}

To find TRIM25-interacting proteins, we performed coIPs of T7-tagged TRIM25 in HeLa cells coupled with quantitative SILAC mass spectrometry. In brief, wildtype T7-tagged TRIM25 was expressed in HeLa cells, and the co-IP was performed using protein A agarose beads coupled with the anti-T7 antibody (Fig. 3a). The control cells (without T7-TRIM25) were grown in 'heavy' medium, and the cells expressing T7-tagged TRIM25 were grown in 'light' SILAC medium. This setup allowed for a quantitative assessment of proteins that bound specifically to TRIM25. After the mass spectrometry analysis, the most enriched protein in the co-IP of T7-TRIM25-expressing cells was TRIM25, which validated our approach (Additional file 2: Table S1). Next, we performed functional Gene Ontology-term annotations for enriched proteins using DAVID [22] and visualized the results in Cytoscape with the Enrichment Map Plugin [23]. Importantly, we found many proteins involved in RNA metabolism such as helicases, RNA processing, nucleotide binding, ribosomal proteins, or proteins involved in RNA stability (Fig. 3a). Recently, a large-scale protein-protein interactome analysis revealed that RNA-binding proteins are likely to coimmunoprecipitate higher numbers of other RNAbinding proteins [24]. Thus, our results emphasize the role of TRIM25 as an RNA-binding protein.

Next, to see which of these protein-protein interactions are mediated by RNA, we performed the same coIP assays using T7-tagged TRIM25 $\triangle$ RBD (Fig. 3b) or T7-tagged TRIM25 but in the presence of RNases (Fig. 3c). In total, 109 proteins were absent in either the T7-TRIM $25 \Delta$ RBD or RNase-treated T7-TRIM25 co-IPs when compared with the wild-type T7-TRIM25 co-IP (Fig. 3d). These proteins, which most likely interact with TRIM25 through RNA, are associated with RNA processing and splicing, RNA stability, RNA transport, and helicase activity. We have validated that a recently identified, physiologically relevant TRIM25 ubiquitination substrate - RNA-binding ZAP [12, 13] - interacts with T7-TRIM25 but not T7-TRIM25 $\triangle$ RBD (Fig. 3e). ZAP has longer and shorter isoforms, which differ by the presence or absence of the poly(ADP-ribose) polymerase domain in the C-terminal region. The binding of both isoforms to TRIM25 was severely reduced upon RNase treatment, proving that the interaction is dependent on the RNA (Fig. 3e). Likewise, DDX5 was predominantly 


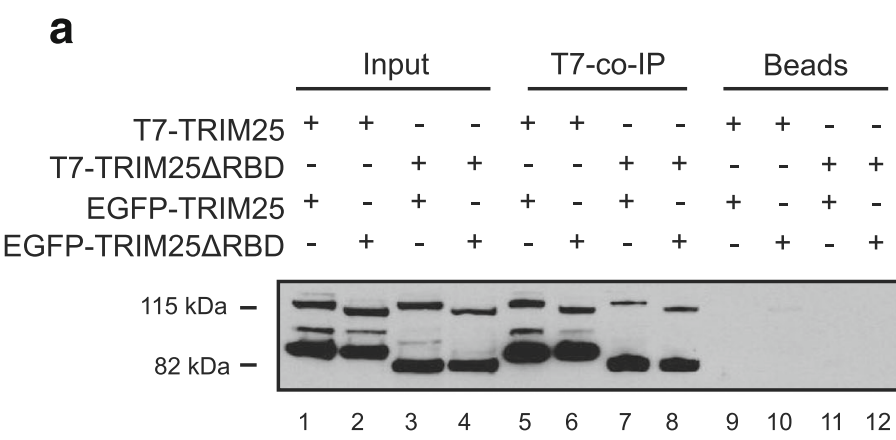

WB: TRIM25

b

\begin{tabular}{|c|c|c|c|c|c|c|c|c|c|c|c|c|}
\hline \multirow[b]{2}{*}{ T7-TRIM25 } & \multicolumn{4}{|c|}{ Input } & \multicolumn{4}{|c|}{ T7-co-IP } & \multicolumn{4}{|c|}{ Beads } \\
\hline & + & - & - & - & + & - & - & - & + & - & - & - \\
\hline T7-TRIM25 $\Delta$ RBD & - & + & - & - & - & + & - & - & - & + & - & - \\
\hline EGFP-TRIM25 & - & - & + & - & - & - & + & - & - & - & + & - \\
\hline GFP-TRIM25 $\Delta$ RBD & - & - & - & + & - & - & - & + & - & - & - & + \\
\hline
\end{tabular}

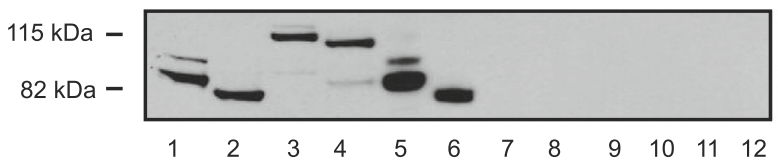

WB: TRIM25

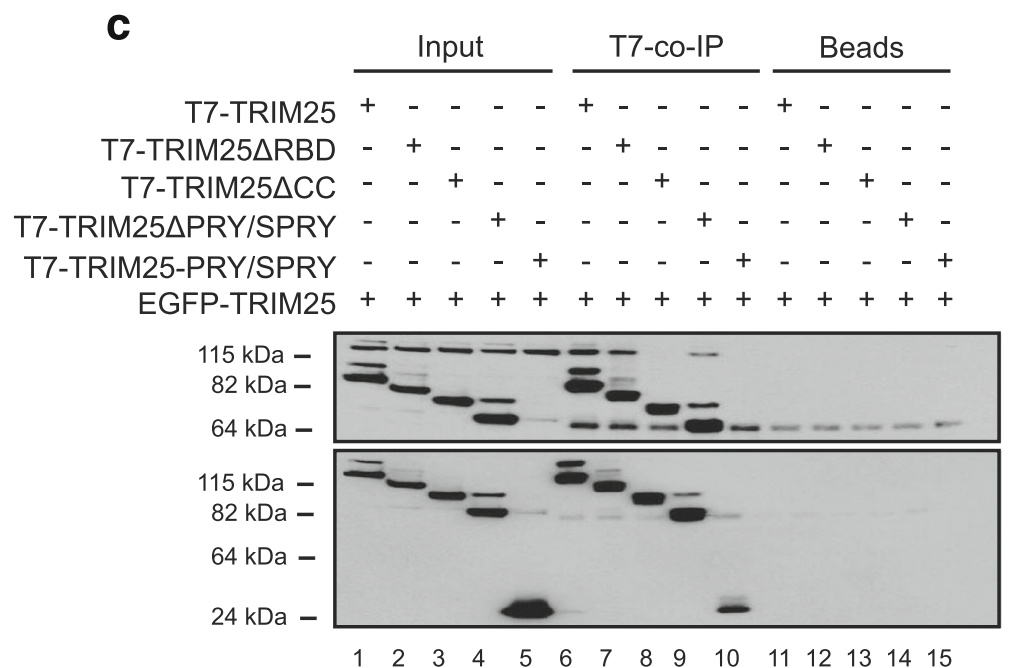

WB: TRIM25

WB: T7

Fig. 2 T7-TRIM25 $\triangle R B D$ supports TRIM25 dimerization. a Co-immunoprecipitation (Co-IP) between T7-tagged TRIM25 and EGFP-tagged TRIM25 in cell extracts prepared from HeLa TRIM25 KO cells overexpressing wild-type or $\triangle$ RBD TRIM25 mutants. Lanes 1-4 represent loading controls. Lanes 5-8 show co-IPs with anti-T7 antibody. Lanes 9-12 represent control co-IPs with IgG antibody bound to protein-A beads. The bound proteins were analyzed by western blotting with anti-TRIM25 antibody. $\mathbf{b}$ Control co-IP shows that CO-IP of EGFP-TRIM25 and EGFP-TRIM25 did not occur with anti-T7 antibody. Lanes 1-4 represent loading controls. Lanes 5-8 show co-IPs with anti-T7 antibody. Lanes 9-12 represent control co-IPs with lgG antibody bound to protein-A beads. The bound proteins were analyzed by western blotting with anti-TRIM25 antibody. c Control co-IP in HeLa TRIM25 KO cell extracts reveals that T7-TRIM25 $\triangle$ RBD and T7-TRIM25 $\triangle$ PRY/SPRY, but not T7-TRIM25 $\Delta C C$ or T7-TRIM25-PRY/SPRY, support dimerization. Lanes 1-4 represent loading controls. Lanes 5-8 show co-IPs with anti-T7 antibody. Lanes 9-12 represent control co-IPs with IgG antibody bound to protein-A beads. The bound proteins were analyzed by western blotting with anti-TRIM25 and anti-T7 antibodies

detected in the co-IP with T7-TRIM25 but not with T7TRIM25 $\triangle$ RBD, although the RNase treatment resulted in only a decreased interaction (Fig. 3e). Importantly, many proteins that were enriched in the T7TRIM25 $\triangle$ RBD and RNase-treated T7-TRIM25 assays were associated with ribosomal and translation functions
(Fig. 3b, c), representing RNA-independent interactions with TRIM25 (Additional file 2: Table S1). Surprisingly, the T7-TRIM25 $\triangle$ RBD and RNase-treated T7-TRIM25 co-IPs detected several proteins that were not seen in the wild-type T7-TRIM25 co-IP, likely due to a synthetic effect of the mutation, lack of binding to RNA, or 
a

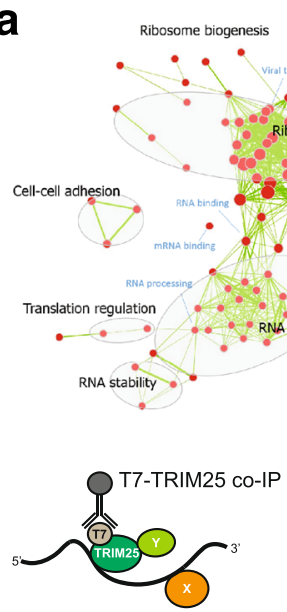

b

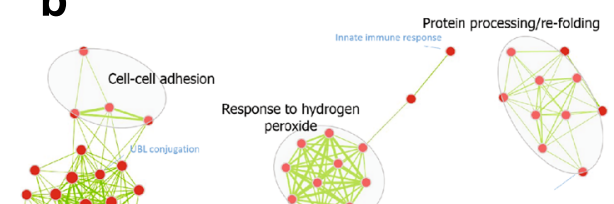

60 Helicase

$\because \cdots$

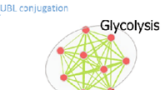

...

- $60^{\circ}$

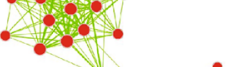

..

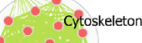

$\because \because \cdots$

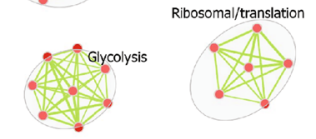

$\therefore$

T7-TRIM25ARBD co-IP
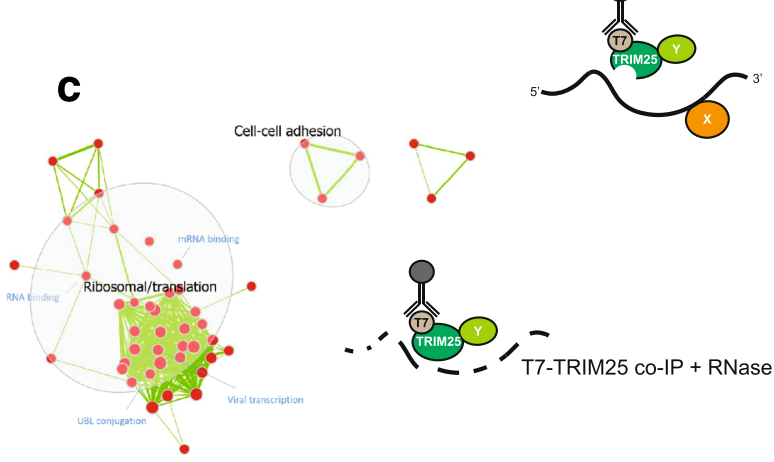

T7-TRIM25 co-IP + RNase

d

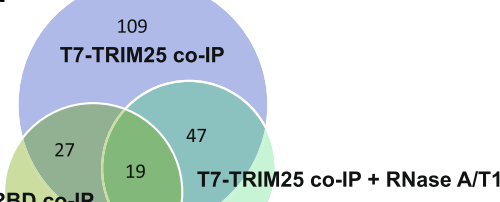

T7-TRIM25 $\triangle R B D$ CO-IP

24

\section{e}
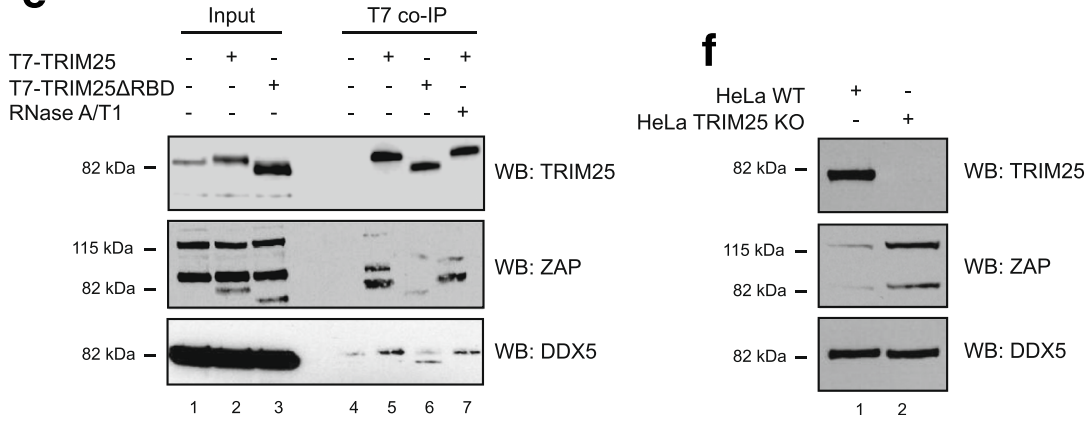

Fig. 3 SILAC combined with TRIM25 co-immunoprecipitation (Co-IP) reveals a network of associated RNA-binding proteins. a A diagram of a co-IP experiment with T7-tagged TRIM25 in the presence of endogenous RNAs together with Gene Ontology enrichment map of proteins coimmunoprecipitated with T7-TRIM25. Enriched protein sets are represented as nodes (red circles) connected by edges (green lines), with the size of nodes and edges representing the number of proteins in gene sets and amount of overlap. $\mathbf{b}$ A diagram of a co-IP experiment with T7-tagged TRIM25 $\triangle$ RBD in the presence of endogenous RNAs accompanied by the Gene Ontology enrichment map of proteins co-immunoprecipitated with T7-TRIM25 $\triangle$ RBD. c A diagram of a co-IP experiment with T7-tagged TRIM25 in the presence of RNases together with Gene Ontology enrichment map of proteins co-immunoprecipitated with T7-TRIM25 in the presence of RNases. $\mathbf{d}$ Venn diagram representing numbers of overlapping proteins identified in the co-IPs with T7-TRIM25, T7-TRIM25 $\triangle$ RBD, and T7-TRIM25 in the presence of RNases. e Validation of co-IP experiments show western blot against ZAP and DDX5 proteins. ZAP has two isoforms co-immunoprecipitated by T7-TRIM25 but not by T7-TRIM25 $\triangle$ RBD or T7-TRIM25 in the presence of RNases. Note that anti-ZAP antibody is picking up a signal from overexpressed TRIM25. DDX5 binding to TRIM25 is partially RNA-dependent as co-IP by T7-TRIM25 in the presence of RNases decreases but does not eliminate its interaction with TRIM25. Lanes 1-3 represent loading controls. Lane 4 shows control co-IPs in mock HeLa cell extracts. Lanes 5-7 show anti-T7 co-IP. f Western blot analysis of wild-type (WT) and TRIM25 knock-out (KO) HeLa cells shows upregulation of ZAP protein in the KO cells. DDX5 levels do not change and serve as a loading control 
unspecific binding events. Altogether, these results reaffirm that the PRY/SPRY domain is important for RNA-binding and show that TRIM25 interacts with many RNA-binding proteins, using RNA as a scaffold.

We set out to find out if TRIM25 KO results in global perturbation of protein levels. To do so, we used SILAC mass spectrometry on the wild-type and TRIM25 KO HeLa cells. Our results showed that the levels of only a few proteins were affected more than two-fold (Additional file 3: Table S2). Among the affected proteins we found ZAP, which was substantially upregulated in TRIM $25 \mathrm{KO}$ cells when compared with the wild-type cells. Western blot analysis of the wild-type and $\mathrm{KO}$ cells validated this observation (Fig. 3f). To see if TRIM25-dependent proteasome degradation of ZAP contributes to its lower levels in the wild-type cells we treated the cells with proteasome inhibitor MG132. Upon MG132 treatment, we noticed an increase in ZAP levels only in the wild-type cells but not in the TRIM25 KO cells (Additional file 1: Figure S2c). Conversely, the signal corresponding to ubiquitin was elevated in both wild-type and TRIM25 $\mathrm{KO}$ cells (Additional file 1: Figure S2d). Importantly, overexpression of T7-TRIM25, but not of T7-TRIM25 $\triangle R B D$ or catalytically inactive T7-TRIM25 $\Delta$ RING, decreased the levels of ZAP in TRIM25 KO cells (Additional file 1: Figure S2e), demonstrating that TRIM25-mediated proteasome degradation of ZAP contributes to its normal levels. Furthermore, these results suggest that the functional interaction between TRIM25 and ZAP is RNA dependent.

\section{CLIP-seq reveals an extensive TRIM25 RNA interactome}

To detect endogenous RNA substrates of TRIM25, we performed UV crosslinking and immunoprecipitations in HeLa cells transiently expressing T7-tagged TRIM25, followed by high-throughput sequencing of TRIM25associated RNAs (CLIP-seq). After UV crosslinking and immunoprecipitation, we observed a specific RNAprotein complex (Additional file 1: Figure S3a). CLIP-seq of T7-TRIM25 $\triangle$ RBD resulted in a lack of detectable RNA-protein interactions (data not shown). The RNA from the T7-TRIM25/RNA complex was isolated using a CLIP-seq protocol and sequenced on a HiSeq platform. Data analysis and cluster identification using pyCRAC [25] revealed a significant correlation between three biological replicates, with a correlation coefficient between clusters from 0.94 to 0.96 (Additional file 1: Figure S4). A total of 5549 clusters were common between all three experiments, and we identified 2611 distinct transcripts (Additional file 4: Table S3). Importantly, there was no correlation between transcript abundance, measured by a separate RNA-seq assay, and the cluster intensities, with a Pearson correlation of 0.1 (Additional file 1:
Figure S5), showing that TRIM25 does not simply bind the most abundant RNAs but has a substrate preference. Among the identified transcripts, mRNAs and lincRNAs were the most abundant (Fig. 4a). Gene Ontologyterm annotation of TRIM25-bound transcripts revealed several nodes, including RNA processing and translation, WD repeats, phosphorylation, and ubiquitination (Additional file 1: Figure S6a).

To determine the TRIM25 binding sequence preference, we used the pyMotif program from the pyCRAC package and WebLogo on identified clusters to search for enriched k-mers. While no single strong consensus motif was identified, we found that TRIM25 prefers Gand $\mathrm{C}$-rich sequences (Fig. 4b). This was confirmed by a bioinformatics analysis showing a significantly higher GC content in TRIM25 clusters compared to random transcripts or random exons ( $P$ values for both comparisons were $2.2 \times 10^{-16}$ ) (Fig. 4c). When we clustered our consensus motifs we found some discrete, G- and C-rich short motifs (Additional file 1: Figure S6b). In the future, it will be interesting to check if any of these motifs contribute towards TRIM25's RNA-binding specificity. Next, we assessed the distribution of TRIM25 binding in mRNAs. This revealed that most binding sites are in exons (56\%), with the 3 '-UTR also prominently represented (23\%) (Fig. 4d). Furthermore, we observed a noticeable enrichment of TRIM25 clusters just after the stop codon (Fig. 4e). TRIM25 binds to its own transcript, which could be biologically important (Additional file 1: Figure S3b). Intriguingly, even though TRIM25 is localized to both nucleus and cytoplasm [14], intron binding events were not common in CLIP-seq analysis; this could be demonstrated on TRIM25 mRNA target cMyc. Both high throughput CLIP-seq and TRIM25 RNA immunoprecipitation (RIP) followed by targeted amplification of c-Myc transcript showed that TRIM25 predominantly interacts with mature mRNA (Additional file 1: Figure S3c, d). Nevertheless, TRIM25 interacts with many RNA-binding proteins (Fig. 3) and they could provide a platform for interactions with other targets at the level of pre-mRNAs.

To validate the CLIP-seq experiments, we performed RIP followed by qRT-PCR of selected TRIM25 targets in HeLa cells overexpressing either wild-type T7-TRIM25 or T7-TRIM25 $\triangle$ RBD (Fig. 4f, g). RIP qRT-PCR with the wild-type TRIM25 showed significant enrichment of tested mRNAs (Fig. 4f) and miRNAs (Fig. 4g). Intriguingly, the T7-TRIM25 RIP signal on TRIM25 mRNA was much higher than that on other substrates (Fig. 4f). This could not be explained by increased levels of TRIM25 mRNA, which was upregulated only three-fold when compared to the mock-transfected control (data not shown). Importantly, T7-TRIM25 $\triangle$ RBD failed to precipitate any RNAs apart from TRIM25 mRNA itself 
a

TRIM25 CLIP-seq cluster distribution in total RNA

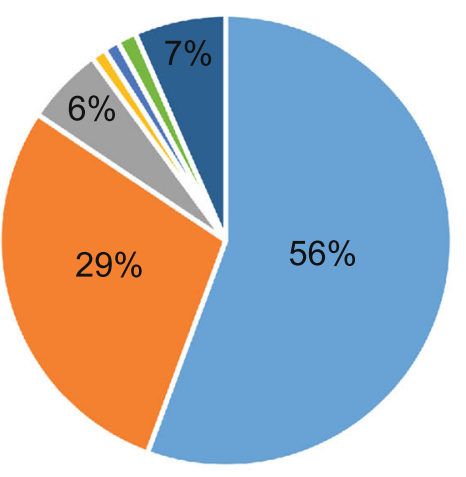

d
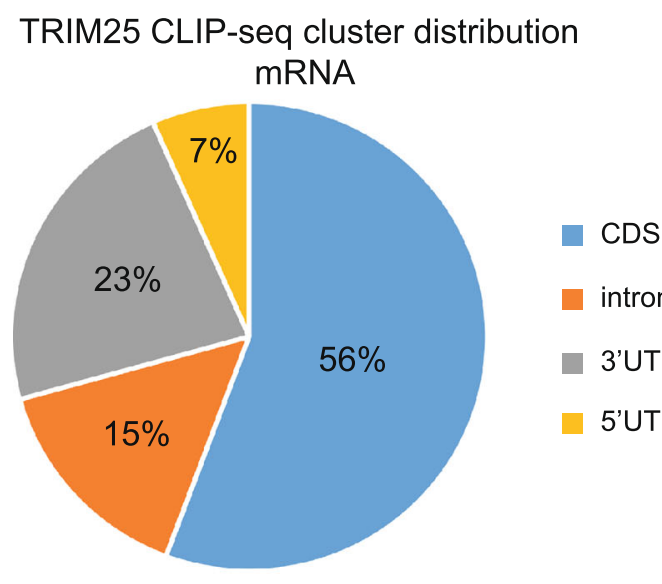

intron

- 3'UTR

- 5'UTR b

TRIM25 binding preference
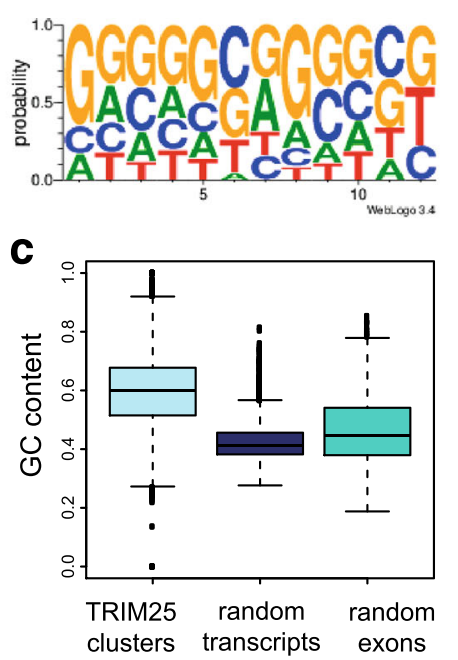

e TRIM25 cluster distribution

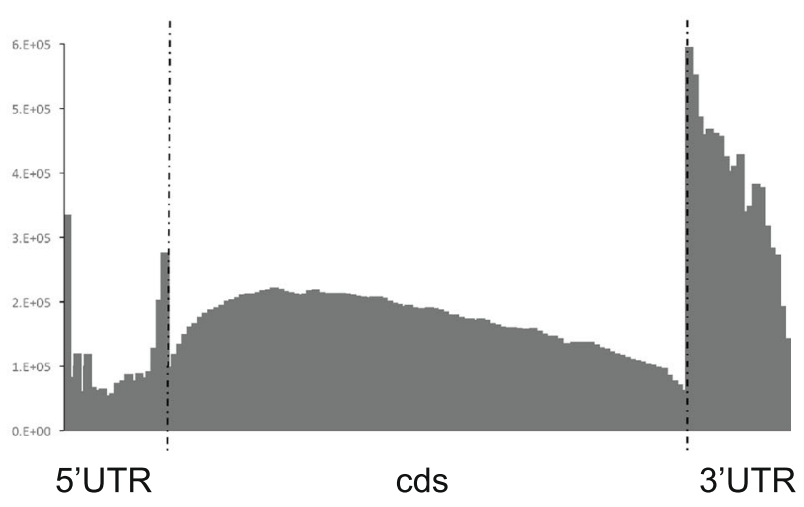

g

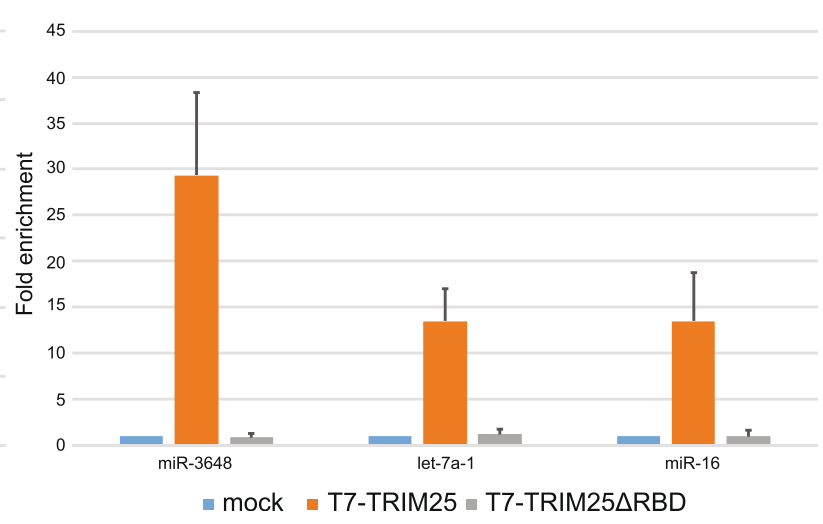

Fig. 4 (See legend on next page.) 


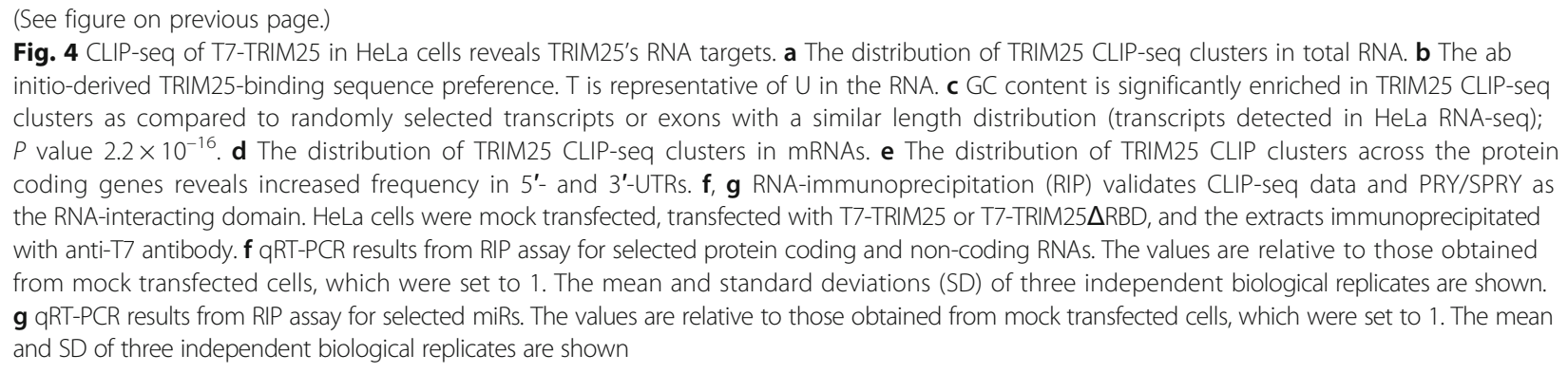

(Fig. 4f). These results could be explained by the binding of the T7 antibody to the nascent TRIM25, which was still associated with polyribosomes and TRIM25 mRNA. As the T7 antibody could co-IP many nascent TRIM25 peptides, which were still associated with the TRIM25 mRNA, the RIP signal would be increased. Altogether, these results uncover the first TRIM25/RNA interactome, suggesting that TRIM25 RNA-related functions may extend well beyond the regulation of pre-let-7 uridylation toward other aspects of RNA metabolism. Finally, these data clearly demonstrate that one of the main TRIM25 targets is its own mRNA.

To gain further insights into TRIM25's role in RNA metabolism we have used qRT-PCR to analyze mRNA levels of selected targets (the same ones as assayed by RIP in Fig. 4f, g) and ZAP mRNA in the wild-type and TRIM25 KO cells. The majority of analyzed transcripts did not display substantial difference between wild-type and KO cells (Additional file 1: Figure S7a, b). ZAP and RNU12 transcripts were moderately upregulated in the TRIM25 KO cells when compared with the wild-type ones. To see if TRIM25 could regulate stability of transcripts we blocked pol II transcription by Actinomycin $\mathrm{D}$ and assayed the levels of selected RNAs by qRT-PCR at various time points (Additional file 1: Figure S7c-e). No statistically significant difference between wild-type and the TRIM25 $\mathrm{KO}$ cells was observed in the stability of ZAP and c-Myc transcripts (Additional file 1: Figure S7c-e), suggesting that the steady-state ZAP mRNA level change seen in the TRIM25 KO cells is due to transcriptional control; this is not surprising as a recent study revealed that TRIM25 is a potent transcriptional regulator [26]. Further in-depth and high throughput experiments will be needed to fully understand the potential role of TRIM25 in RNA metabolism.

\section{Binding of TRIM25 to its mRNA augments TRIM25 ubiquitination}

To uncover the RNA-related function of TRIM25 and the reason behind its binding to its own mRNA, we focused on the well-documented molecular event of TRIM25 K117 autoubiquitination [27]. We confirmed that TRIM25 undergoes autoubiquitination by co- overexpression and co-IP of T7-tagged TRIM25 together with HA-tagged ubiquitin (Additional file 1: Figure S8a, b). This was also confirmed by the in vitro ubiquitination experiments described below. Next, we asked if binding of TRIM25 to its own mRNA augmented TRIM25 ubiquitination. To address this, we overexpressed EGFPTRIM25 or EGFP-TRIM25 $\triangle$ RBD in HeLa cells and assayed for TRIM25 ubiquitination. We used EGFP fusion proteins to distinguish overexpressed and endogenous TRIM25. The overexpression of EGFP-TRIM25 led to the appearance of modified EGFP-TRIM25 (Fig. 5a, b). However, upon EGFP-TRIM25 $\triangle$ RBD overexpression, the intensity of the modified band was noticeably reduced (Fig. $5 \mathrm{a}$, b). Of note, we had to use more EGFP-TRIM25 $\triangle$ RBD plasmid to achieve protein expression comparable to that of EGFP-TRIM25. Surprisingly, overexpression of EGFPTRIM25, but not EGFP-TRIM25 $\triangle$ RBD, led to significant accumulation of modified forms of endogenous TRIM25, with much higher ratios of the modified forms compared to the non-modified form (Fig. 5a, b). These results suggest that the intact RBD is required for efficient ubiquitination and show that endogenous TRIM25 can be modified by EGFP-TRIM25 to a much greater extent than EGFP-TRIM25 modifies itself. One important difference between endogenous TRIM 25 mRNA and the mRNA coding for EGFP-TRIM25 is the presence of 5' - and 3'UTRs, which were shown to bind TRIM25 in the CLIPseq experiments (Additional file 1: Figure S3b).

To determine if the TRIM25 3'-UTR can contribute to ubiquitination of TRIM25, we used a catalytically dead T7-TRIM25 $\Delta$ RING construct, which cannot undergo autoubiquitination, and transfected it into TRIM25 KO cells. We also used a T7TRIM25 $\Delta$ RING_3'-UTR construct that contained the TRIM25 3'-UTR. Notably, we had to use more T7TRIM25 $\Delta$ RING_3'-UTR plasmid to achieve protein expression comparable to that of T7-TRIM25 $\Delta$ RING (Fig. 5c), most likely due to microRNA/3'-UTR-mediated control of protein expression. As predicted, T7TRIM25 $\Delta$ RING does not support efficient autoubiquitination (Fig. 5c). However, upon EGFP-TRIM25 overexpression, we noticed the appearance of ubiquitinated forms of T7-TRIM25 $\Delta$ RING (Fig. 5c, d). We could not 


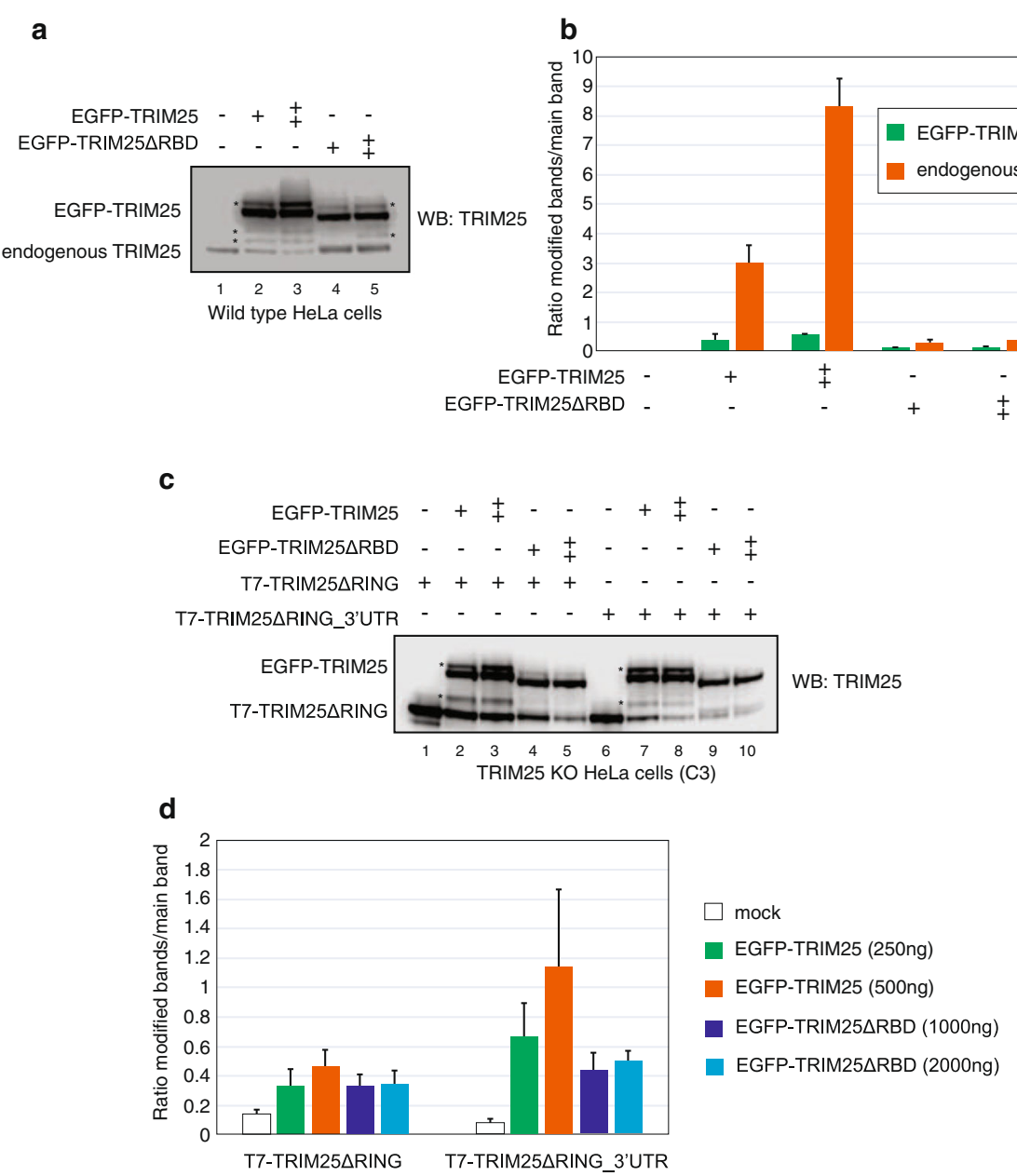

Fig. 5 TRIM25 binding to the RNA augments TRIM25 ubiquitination. a Western blot analysis of HeLa cells transfected with EGFP-TRIM25 or EGFP-TRIM25 $\triangle$ RBD. Lane 1 shows mock transfected control. Lanes 2 and 3 represent results of transfection with increasing amounts of plasmid coding for EGFP-TRIM25 (250 and $500 \mathrm{ng}$ ). Lanes 4 and 5 represent results of transfection with increasing amounts of plasmid coding for EGFPTRIM25 $\triangle$ RBD (1000 and $2000 \mathrm{ng}$ ). b Quantification of western blot results shown in (a). The values show the ratios between the intensity of modified versus main bands. The results are derived from three independent biological replicates and the mean and standard deviations are shown. $\mathbf{c}$ Western

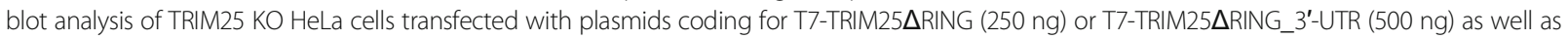

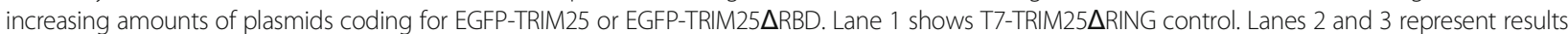
of transfections with T7-TRIM25 RRING and increasing amounts of EGFP-TRIM25 (250 and $500 \mathrm{ng}$ ). Lanes 4 and 5 show results of transfection with T7-

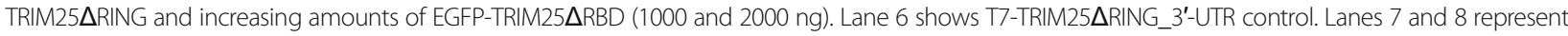
results of transfections with T7-TRIM25 $\Delta$ RING_3'-UTR and increasing amounts of EGFP-TRIM25 (250 and 500 ng). Lanes 9 and 10 show results of transfection with T7-TRIM25 $\Delta$ RING_3'-UTR and increasing amounts of EGFP-TRIM25 RRBD (1000 and 2000 ng). d Quantification of western blot results shown in (c). The values show the ratios between the intensity of modified versus main bands. The results are derived from three independent biological replicates and the mean and standard deviations are shown

detect abundant ubiquitination bands in T7-TRIM25 $\Delta$ RING when EGFP-TRIM25 $\triangle$ RBD was co-over expressed (Fig. 5c, d). Even so, the ratio between the ubiquitinated and main bands still showed a positive value, most likely due to weaker expression of T7TRIM25 $\Delta$ RING in the presence of EGFP-TRIM25 $\Delta$ R $\mathrm{BD}$ and to background noise. Importantly, when we cooverexpressed the T7-TRIM25 $\Delta$ RING_3'-UTR construct with EGFP-TRIM25, we observed a noticeable increase in the ratio of ubiquitinated versus nonubiquitinated bands for TRIM25 $\triangle$ RING, when compared with the T7-TRIM25 $\Delta$ RING construct without the 3'UTR (Fig. 5c, d). To prove that the observed modification of TRIM25 was specific to K117 we performed similar analyses with T7-TRIM25 $\Delta$ RING_K117R constructs. We did not observe modification of T7-TRIM25 $\Delta$ RING_K1 $17 \mathrm{R}$ in the absence or presence of EGFP-TRIM25 (Additional file 1: Figure S8c). Altogether, these results 


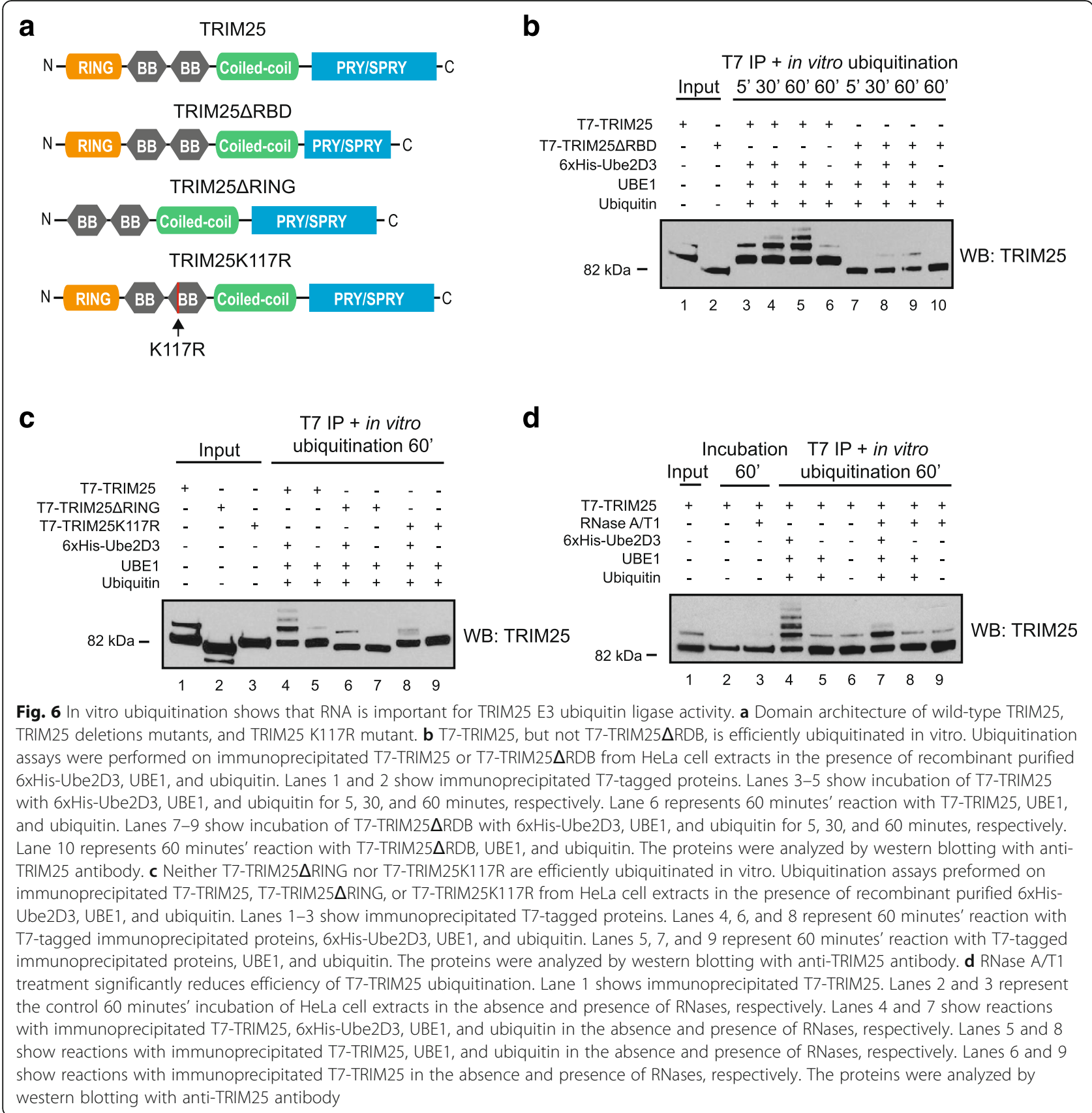

strongly suggest that RNA elements such as the 3 'UTR, as well as TRIM25 binding to the RNA, contribute to TRIM25 ubiquitination.

RNA is necessary for efficient TRIM25 ubiquitination in vitro To test if RNA is necessary for efficient TRIM25 ligase activity, we performed in vitro ubiquitination assays with various TRIM25 constructs (Fig. 6a). Incubation of T7-TRIM25 or T7-TRIM25 $\triangle$ RBD immunoprecipitated from HeLa cells with recombinant proteins from the ubiquitin pathway (Ube2D3, UBE1, and Ubiquitin) revealed efficient, time-dependent ubiquitination of TRIM25 but not TRIM25 $\triangle$ RBD (Fig. 6b). Residual ubiquitination of TRIM25 $\triangle \mathrm{RBD}$ could arise from the direct transfer of ubiquitin from Ube2D3 to the substrate. Next, to show that the observed ubiquitination was specific and dependent on the intrinsic activity of TRIM25, we assayed T7-TRIM25 $\Delta$ RING and T7TRIM25K117R. Neither T7-TRIM25 2 RING nor T7TRIM25K117R was efficiently ubiquitinated compared to wild-type T7-TRIM25 (Fig. 6c), confirming the specificity of the in vitro ubiquitination assay. 
Finally, to see if TRIM25 ubiquitin-ligase activity was dependent on RNA, we performed the ubiquitination assay in the absence or presence of RNase A/ T1. Remarkably, treatment with RNase A/T1 severely inhibited in vitro ubiquitination of T7-TRIM25 (Fig. 6d). Interestingly, RNase A/T1-treated reactions still supported TRIM25 monoubiquitination, likely due to the direct transfer of ubiquitin from Ube2D3 to TRIM25. These results strongly indicate that RNA constitutes an important component of the TRIM25 ubiquitin ligase activity.

\section{Homologous sequences from TRIM proteins rescue the TRIM25 RNA-binding and ubiquitination activities}

To further verify our findings and test whether other TRIM proteins with PRY/SPRY domains could have RNA-binding potential, we prepared constructs encoding chimeric proteins, where the TRIM25 RBD was substituted with homologous sequences from several TRIM proteins (TRIM5, TRIM21, TRIM27, and TRIM65) (Fig. 7a). Moreover, we designed a TRIM25 construct with a scrambled amino acid sequence of the TRIM25 RBD (Fig. 7a). The chimeric TRIM25 protein constructs expressed in HeLa cells showed various levels of modification (Fig. 7b). Importantly, neither T7-TRIM25 $\triangle$ RBD nor T7-TRIM25RBD_src was ubiquitinated (Fig. 7b). To see if these results translated to RNA-binding efficiency, we performed RNA pull-down assays with pre-let-7a-1 in HeLa cell extracts. Control assays confirmed that T7-TRIM25, but not T7-TRIM25 $\triangle$ RBD, could bind RNA (Fig. 7c). All chimeric TRIM25 proteins, apart from T7-TRIM25 $\triangle$ RBD_src, showed efficient binding to RNA (Fig. 7d, e). Notably, we did not observe modified bands for TRIM25 in the RNA pull-down assays because the total protein amounts loaded on the gel were one order of magnitude lower than those in the overexpression experiments. To our surprise, there was a strong positive correlation (correlation coefficient $0.87, P=0.01$ ) between protein modification and RNA pull-down efficiencies (Fig. 7f). Altogether, these results imply that RNA-binding is an important element in TRIM25 ubiquitination efficiency and suggest that many other TRIM proteins with the PRY/ SPRY domain could harbor, as yet uncharacterized, RNAbinding potential.

\section{RNA is necessary for efficient ubiquitination of ZAP by TRIM25 in vitro}

To test if RNA was necessary for efficient ubiquitination of ZAP, a physiologically important TRIM25 target, we performed in vitro ubiquitination assays of ZAP (ZAP short) with the TRIM25 constructs described earlier (Fig. 6a). Incubation of T7-ZAP with T7-TRIM25 or T7TRIM25 $\triangle$ RBD immunoprecipitated from $\mathrm{HeLa}$ cells with recombinant proteins from the ubiquitin pathway revealed efficient time-dependent ubiquitination of ZAP by TRIM 25 but not TRIM $25 \triangle$ RBD (Fig. 8b). Notably, to see efficient ubiquitination of ZAP we had to use higher concentrations of recombinant proteins from the ubiquitin pathway than those used for TRIM25 autoubiquitination experiments. As a result, TRIM25 autoubiquitination was much more pronounced in experiments with ZAP (Fig. 8a) than seen in the case of TRIM25only assays (Fig. 6b). Furthermore, it has been recently reported that ZAP enhances TRIM25 autoubiquitination [13]. Next, to show that the observed ZAP ubiquitination was specific and dependent on the activity of TRIM 25, we assayed T7-TRIM25 $\Delta$ RING and T7-TRIM25K 117R. TRIM25 $\Delta$ RING but not T7-TRIM25K117R lost the ability to ubiquitinate T7-ZAP (Fig. 8b). Intriguingly, we observed autoubiquitination of T7-TRIM25K117R, which could be explained by increased concentrations of the proteins from the ubiquitination pathway and ZAP stimulatory activity towards TRIM25 [13]. Finally, to see if TRIM25 E3 ubiquitin ligase activity towards ZAP was dependent on RNA, we performed the ubiquitination assay in the absence or presence of RNase A/T1. Importantly, the treatment with RNase A/T1 severely inhibited in vitro T7-ZAP ubiquitination by T7-TRIM25 (Fig. 8c). These results strongly indicate that TRIM25 uses RNA as a scaffold for efficient ubiquitination of its protein targets.

\section{Discussion}

TRIM25 E3 ubiquitin ligase is a new RNA-binding protein with a role in Lin28a-mediated uridylation of pre-let-7 transcripts [14]. Here, we revealed that the RNA-binding activity of TRIM25 is contained in its PRY/SPRY region. We also presented the first highthroughput analysis of the molecular interactomes of TRIM25. We showed that TRIM25 is a bona fide RNAbinding protein associated with many proteins involved in RNA metabolism and that interacts with numerous coding and non-coding transcripts. This suggests that TRIM25 could play a role in the regulation of RNA metabolism. Our data showed that TRIM25 binding to RNA, and to the 3'-UTR of TRIM25 mRNA in particular, can augment its autoubiquitination. Finally, we demonstrated that RNA is an important constituent of TRIM25's ubiquitin ligase activity towards itself and its physiologically relevant protein target ZAP. Recently, ZAP was shown to suppress HIV-1 replication by binding CG-rich regions in viral RNAs [28]. Intriguingly, the first paper that described ubiquitin conjugation to substrates showed that tRNA is required for selected ubiquitination reactions [29]. We propose a model whereby the ubiquitin ligase activity of TRIM25 is substantially enhanced by using RNA as a scaffold (Fig. 9a). Alternatively, binding of TRIM25 to RNA could enhance its 


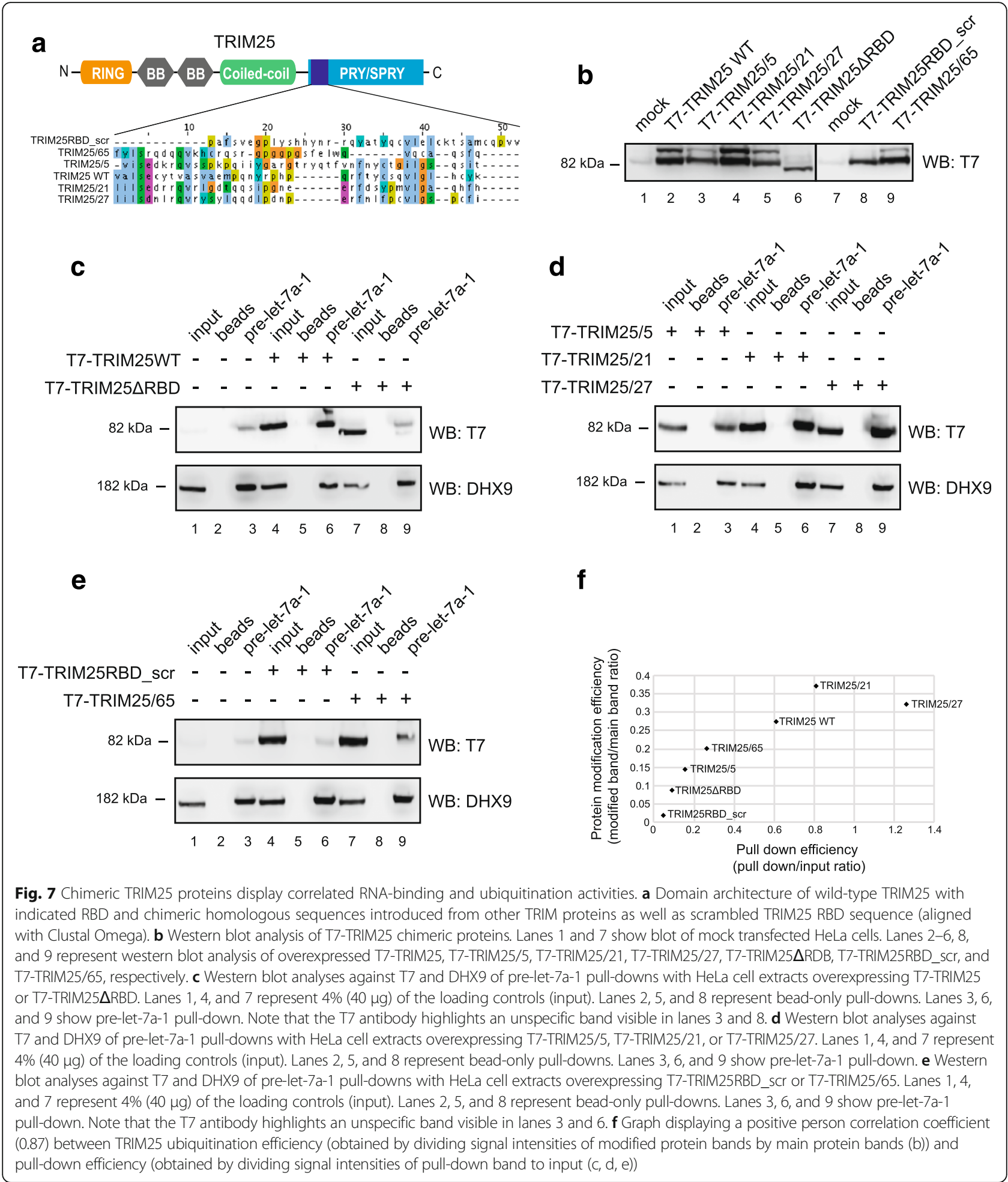

ubiquitin ligase activity by allosteric changes (Fig. 9b). These models should be verified by detailed structural analysis of the free and RNA-bound TRIM25. Finally, it is exciting to speculate that activity of many other E3 ubiquitin ligases could depend on binding to RNA.
Our results carry very important functional implications given that the 3 '-UTRs are platforms for regulatory RNA-binding proteins and that the lengths of the 3 '-UTRs associated with specific mRNAs are variable. For example, cancer cells have mRNAs with much 


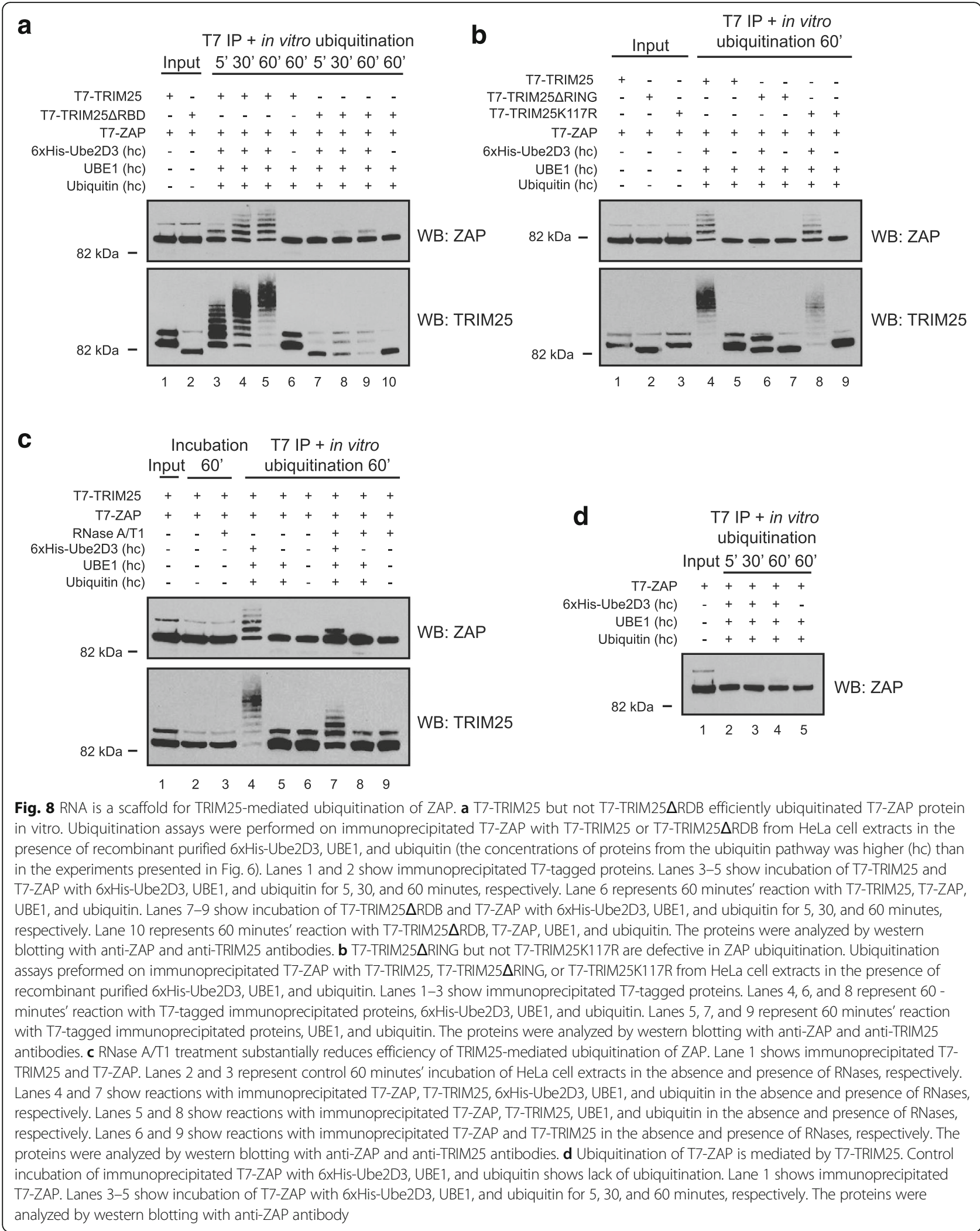




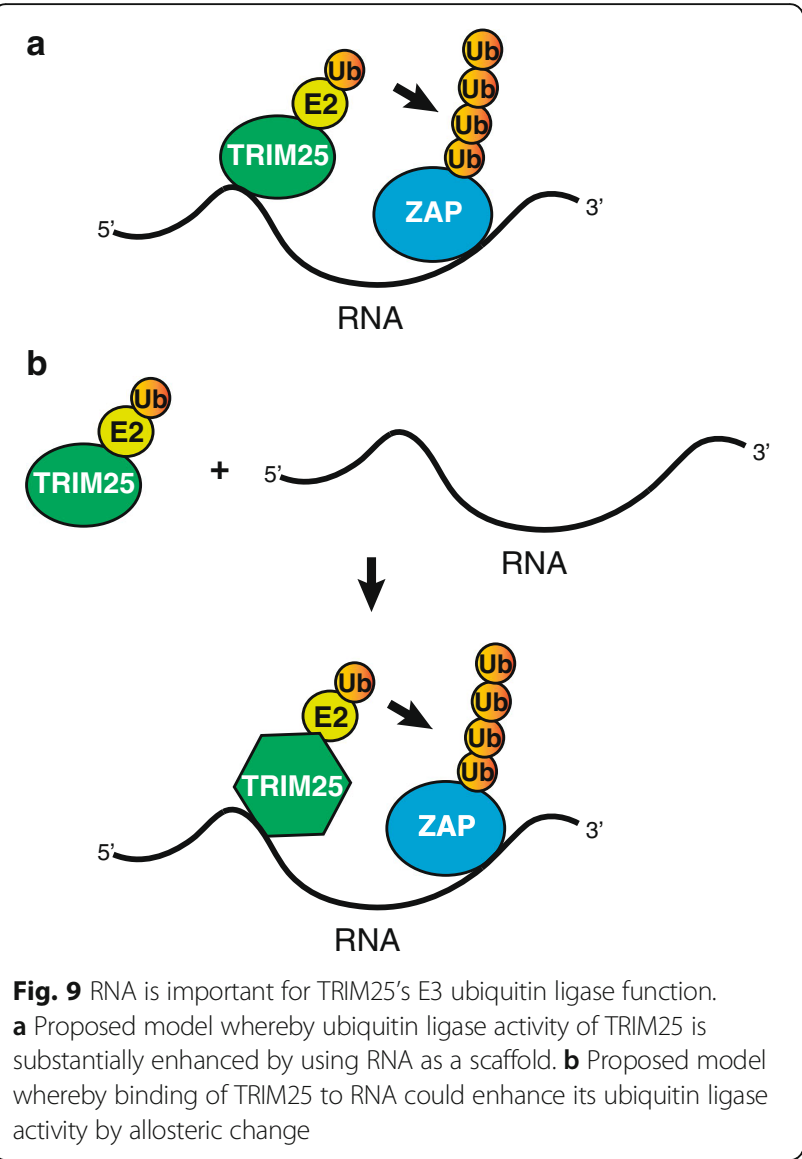

shorter 3 '-UTRs, which exhibit increased stability and produce more proteins in part through the loss of microRNA-mediated repression [30]. Additionally, the shortening of 3 '-UTRs is associated with proliferating $\mathrm{T}$ cells but is not accompanied by a corresponding change in protein levels [31]. For these reasons, regulation of the lengths of the 3'-UTRs could allow for tight control of the type and amount of TRIM25 that is associated with specific mRNAs and the subsequent ubiquitination/ ISGylation of nascent peptides. This could be an important mechanism in the physiological and pathological control of protein stability and function. Importantly, TRIM25 also binds to the 5'-UTRs of mRNAs and within coding regions. Thus, the RNA-related functions of TRIM25 might not be limited to 3'-UTRs, which could result in different functions depending on where in the RNA the TRIM25 binds.

Previously, the CC domain of TRIM25 has been implicated in binding to RNA [3]. TRIM25 deletion mutants lacking the CC domain were unable to interact with RNA in the UV immunoprecipitation and T4 polynucleotide kinase assays [3]. In contrast, the mutant lacking the PRY/SPRY domain still retained its RNAbinding ability. Surprisingly, the results from the proteome-wide analysis of RNA-binding domains in
HeLa cells (Fig. 1b) [19], as well as the results obtained from the RNA pull-down (Fig. 1e), EMSA (Fig. 1a, c), co-IP (Fig. 3), and RIP (Fig. 4f, g) assays, implicate the PRY/SPRY domain as the point of contact with the RNA. This discrepancy could arise from the fact that deletion of the CC domain from TRIM25 eliminates its dimerization $[15,17,18]$ and oligomerization [16] ability (Fig. 2c). While it is not univocally confirmed that TRIM25 needs to dimerize to bind RNA, such a phenomenon is widespread across many RNA-binding proteins $[20,21]$. Additionally, the experiments presented by Kwon et al. [3] were performed in wild-type mouse embryonic stem cells, where levels of endogenous TRIM25 are particularly high. This means that any ectopically overexpressed TRIM25 mutant (if it has the CC domain), including TRIM25 $\triangle$ PRY/SPRY, will have a chance to heterodimerize with the wild-type, endogenous TRIM25, which could mask the requirements for a TRIM25 domain, such as PRY/SPRY, to bind to RNA. Our RNA pull-down data with the extracts from the TRIM25 KO cells clearly demonstrated that T7TRIM25 $\triangle$ PRY/SPRY could not bind RNA (Fig. 1g). Further structural characterization of the TRIM25/RNA complex should reveal detailed features of this interaction and shed more light on this novel, putative RNAbinding domain.

Importantly, there are more than 80 TRIM proteins in the human genome [5], divided into 11 subfamilies based on their structural composition; 43 of these have PRY/SPRY domains (subfamilies C-I and C-IV). Although there is a high degree of similarity between them, only TRIM25 (from TRIM proteins containing PRY/ SPRY) was identified in HeLa [1] and mouse embryonic stem cell [3] screens for proteins that bind to RNAs, which could reflect a lack of saturation of RNA-capture screens or lack of expression of many other PRY/SPRYcontaining TRIM proteins in the cell lines used for these assays. Interestingly, another protein from the TRIM family, TRIM71, was shown to use its NHL, but not its CC, domain to bind RNA [3, 32]. Our results from the RNA pull-down experiments with chimeric TRIM25/ TRIM5, TRIM25/TRIM21, TRIM25/TRIM27, and TRIM25/TRIM65 proteins indicate that other TRIM proteins with the PRY/SPRY domain could harbor yet undiscovered RNA-binding potential (Fig. 7). Thus, in the future, it will be important to assay other TRIM proteins to determine their RNA-binding activities. Notably, other E3 ubiquitin ligases, including Mex3b and Dizip3, have been shown to use RNA as a scaffold to mediate the ubiquitination of their substrates, which are bound to the same RNA [33]. Roquin is another example of an E3 ubiquitin ligase with a well-established RNA-binding ability [34]. Moreover, the BRACA1 E3 ubiquitin ligase, which is implicated in familial breast cancer, has been 
shown to interact with RNAs [35]. Altogether, these results strongly suggest the possibility of widespread roles of E3 ubiquitin ligases in the direct regulation of RNA metabolism.

TRIM25 is involved in normal development and disease [36]. For example, TRIM25 has been implicated in estrogen-dependent organ development [37]. Consequently, TRIM25 $\mathrm{KO}$ mice present significantly underdeveloped uteri [38, 39]. Furthermore, TRIM25 plays a role in promoting proliferation of many cancer cell types, often in association with the estrogen response [40-43]. Recently, TRIM25 has been shown to be a master regulator of breast cancer metastasis [26]. Additionally, TRIM25 is known for its role in the RIG-I-mediated interferon pathway $[5,8]$. Upon detection of viral RNAs with 5'-ppp-RNA, RIG-I activates a signaling cascade that results in phosphorylation-mediated dimerization of the transcription factor IRF3, leading to type I interferon (interferon $\alpha$ and $\beta$ ) expression $[9,10]$. K63-linked ubiquitination of RIG-I by TRIM25 is required for an efficient interferon response [11]. RIG-I can also be stimulated by unanchored polyubiquitin chains generated by TRIM25 [44]. This interaction is targeted by viruses to suppress the interferon response. For example, the influenza's RNA-binding protein NS1 inhibits TRIM25-mediated ubiquitination of RIG-I by binding to the TRIM25 CC domain and disrupting its dimerization, allowing the virus to avoid the innate immune response [45]. This inhibitory activity is significantly reduced in an NS1 mutant, R38A/K41A, which cannot bind RNA [46], raising questions about a possible functional link between the RNA-binding activities of TRIM25 and NS1. Finally, the SARS-CoV nucleocapsid protein (Nprotein) has been shown to inhibit TRIM25 by binding to TRIM25's PRY/SPRY domain [47]. Considering our results, it is plausible that N-protein could directly inhibit the TRIM25 RNA binding activity. To fully understand the RNA-related role of TRIM25 in development and disease, it will be important to characterize TRIM25/RNA complexes, especially in the context of RIG-I activation by viral RNAs.

\section{Conclusions}

In summary, we have presented the transcriptome and proteome-wide interactomes of a novel RNA-binding protein, TRIM25. We showed that the PRY/SPRY domain is responsible for the ability of TRIM25 to bind RNA. Finally, we have provided evidence that RNA is an important component in TRIM25's ubiquitin ligase activity. A recent genome-wide bioinformatics screen of the human proteome identified more than 2600 proteins with RNA-binding capacity [48]. Unravelling the RNArelated characteristics and functions of these proteins, including TRIM25, will undoubtedly reveal novel biologically and medically relevant molecular pathways.

\section{Methods \\ EMSA}

EMSAs were performed with an end-labeled pre-let-7a-1 probe $\left(50 \times 10^{3}\right.$ counts per minute, $\left.\sim 0.1 \mathrm{pmol}\right)$ and the indicated amounts of proteins produced in E. coli. Binding reactions were performed in $16-\mu \mathrm{L}$ reactions containing gel exclusion buffer (4.8 mM Tris ( $\mathrm{pH}$ 8), $144 \mathrm{mM} \mathrm{NaCl}, 0.96 \mathrm{mM}$ DTT) supplemented with $3 \mathrm{mM} \mathrm{MgCl}_{2}, 0.5 \mathrm{mM}$ ATP, and $37.5 \mathrm{mM}$ creatine phosphate. Reactions were incubated at $4{ }^{\circ} \mathrm{C}$ for $1 \mathrm{~h}$, followed by electrophoresis on a $6 \% \mathrm{w} / \mathrm{v}$ non-denaturing polyacrylamide gel. The signal was recorded with radiographic film or as exposed to a phosphoimaging screen and scanned on a FLA-5100 scanner (Fujifilm).

\section{RNA pull-down assay}

RNA pull-down assays were performed as previously described [49], with slight modifications. Briefly, total protein extracts were incubated with in vitro-transcribed RNAs that were chemically coupled to beads [50]. The incubation was followed by a series of washes with buffer G $(20 \mathrm{mM}$ Tris ( $\mathrm{pH} 7.5), 135 \mathrm{mM} \mathrm{NaCl}, 1.5 \mathrm{mM}$ $\mathrm{MgCl}_{2}, 10 \%$ v/v glycerol, $1 \mathrm{mM}$ EDTA, $1 \mathrm{mM}$ dithiothreitol, and $0.2 \mathrm{mM}$ phenylmethanesulfonyl fluoride). After the final wash, the proteins associated with the beads were analyzed by western blotting.

\section{Co-immunoprecipitation}

Extracts prepared from HeLa cells transfected with plasmids expressing T7-tagged or EGFP-tagged proteins were incubated with protein A agarose with the anti-T7 antibody. The bound proteins were separated on a 4$12 \%$ SDS polyacrylamide gel and analyzed by western blotting. As indicated, some samples were treated with RNases A and T1 prior to loading on the gel. For mass spectrometry, the T7-bound beads were incubated with HeLa extracts grown in Light or Heavy R6K4 (13C-labeled arginine and 2D-labeled lysine) isotopes (Dundee Cell Products LM014/16). Next, the bound proteins were run on a $4-12 \%$ SDS polyacrylamide gel followed by in-gel digestion as described previously [49]. Gene Ontology enrichment mapping was performed by functionally annotating the enriched proteins using DAVID [22] and visualizing them with the Enrichment Map Plugin v2.1.0 [23] in Cytoscape ( $P$ value cutoff $=0.001$; false discovery rate $\mathrm{Q}$-value cutoff $=0.1$; Jaccard coefficient $=$ $0.25)$. Enriched protein sets are represented as nodes (red circles) connected by edges (green lines), with the sizes of nodes and edges representing the number of proteins in gene sets and the amount of overlap. 


\section{CLIP-seq}

The protocol was adapted from Moore et al. [51] using adapters and PCR oligonucleotides described by Kilchert et al. [52]. Briefly, two P100 dishes per sample were transfected with plasmid expressing T7-tagged TRIM25. Anti-T7 antibody was coupled to $50 \mu \mathrm{L}$ of Dynabeads per sample. Following immunoprecipitation, the RNA was dephosphorylated, the 3 ' linker was ligated and radiolabeled, and the $5^{\prime}$ linker was ligated on the beads. The 3' linker reaction was performed in a total of $80 \mu \mathrm{L}$, with $1 \times$ PNK buffer, $1 \mu \mathrm{M} 3$ ' adapter (3' adapter in CLIP oligos), $800 \mathrm{U}$ T4 RNA ligase 2 truncated K227Q, 80 U RNase out, and 10\% PEG 8000. The 5' linker reaction occurred in a total of $80 \mu \mathrm{L}$, with $1 \times$ PNK buffer, $1 \mathrm{mM}$ ATP, $12.5 \mathrm{nM} 5{ }^{\prime}$ linker L5B (L5Ba-d in CLIP oligos), $80 \mathrm{U}$ RNase OUT, and $40 \mathrm{U}$ T4 RNA ligase. The RNA was reverse transcribed (RT Oligo in CLIP oligos) and amplified by PCR (P5_Forward and P3_barcode Reverse $\mathrm{BC} 1$ or $\mathrm{BC} 2$ ) using LA Takara Taq. The libraries were analyzed on an Illumina Miseq system, and the data were processed using the pyCRAC software [25]. TRIM25 binding motifs were analyzed using pyMotif and WebLogo on identified clusters. The distribution of TRIM25 CLIP clusters across the protein coding genes was performed using pyBinCollector. The correlation between the experiments was calculated by comparing the heights of the clusters in the three experiments. The correlation with total RNA abundance was performed by comparing the heights of the clusters common in all three experiments with the transcript abundance in the RNA-seq data derived from HeLa cells.

\section{RIP}

The RIP experiments were performed as in the CLIP-seq immunoprecipitations, except for cross-linking and RNase treatment. The bound RNAs were isolated by Trizol extraction, and RNAs were identified by qRTPCR, using the SuperScript III Platinum SYBR Green One-Step qRT-PCR Kit (Life Technologies) following the manufacturer's instructions on a Roche 480 LightCycler.

\section{In vitro ubiquitination}

Immunoprecipitation was performed as described above. Following the final wash, proteins bound to beads were incubated with Ube1, His-Ube2D3, and ubiquitin (UBPBio), where $1 \mathrm{mg} / \mathrm{mL}$ of Ube1, $2 \mathrm{mg} / \mathrm{mL}$ of His-Ube2D3, and $50 \mathrm{mg} / \mathrm{mL}$ of ubiquitin were used for TRIM25 autoubiquitination and $5 \mathrm{mg} / \mathrm{mL}$ of Ube1, $10 \mathrm{mg} / \mathrm{mL}$ of HisUbe2D3, and $250 \mathrm{mg} / \mathrm{mL}$ of ubiquitin were used for ZAP ubiquitination. The reactions were carried out at $37{ }^{\circ} \mathrm{C}$ for 1 hour or as otherwise indicated and were stopped by addition of loading buffer and reducing agent. The protein ubiquitination was analyzed by western blot with anti-TRIM25 or anti-ZAP antibodies.

\section{Western blot}

Total protein samples were resolved by standard NuPAGE SDS-PAGE electrophoresis with MOPS running buffer (Life Technologies) and transferred onto a nitrocellulose membrane. The membrane was blocked overnight at $4{ }^{\circ} \mathrm{C}$ with $1: 10$ western blocking reagent (Roche) in Tris-buffered saline buffer with $0.1 \%$ Tween20 (TBST). The following day, the membrane was incubated for 1 hour at room temperature with the primary antibody solution in 1:20 western blocking reagent diluted in TBST. Antibodies used included rabbit polyclonal anti-TRIM25 (1:1000, ab167154, clone EPR7315, Abcam), rabbit polyclonal anti-DHX9 (1:1000, 17721-1AP, RRID - AB_2092506, Protein-Tech), mouse monoclonal anti-tubulin (1:10000, T6199, RRID - AB_477583, Sigma-Aldrich), mouse monoclonal anti-T7 tag HRP conjugate $(1: 10000,69048$, RRID - AB10807495, Novagen), mouse monoclonal anti-HA-probe HRP conjugate (1:1000, sc-7392HRP, F-7, Santa Cruz), rabbit monoclonal anti-DDX5 (1:1000, ab126730, RRID - AB_111 30291, Abcam), and rabbit polyclonal anti-ZAP (ZC3HAV1) (1:1000, 16820-1-AP, Protein-Tech). After washing in TBST, the blots were incubated with the appropriate secondary antibody conjugated to horseradish peroxidase and detected with the SuperSignal West Pico detection reagent (Thermo Scientific) or WesternSure ECL substrate (Licor). The membranes were stripped using the ReBlot Plus Strong Antibody Stripping Solution (Chemicon) equilibrated in water, blocked in 1:10 western blocking solution in TBST, and re-probed as described above.

Cell culture, overexpression, and actinomycin D treatment HeLa cells (American Type Culture Collection) were maintained in standard Dulbecco's modified Eagle's medium (DMEM) (Life Technologies), supplemented with $10 \%$ fetal bovine serum (Life Technologies). For SILAC mass spectrometry, cells were cultured in DMEM supplemented with Light or Heavy R6K4 $\left({ }^{13} \mathrm{C}\right.$-labeled arginine and ${ }^{2} \mathrm{D}$-labeled lysine) isotopes (Dundee Cell Products LM014/16). Fragments encoding proteins of interest were cloned into the pCG T7 plasmid and transiently expressed in HeLa cells using Lipofectamine 2000 (Invitrogen). For RNA stability assays, $10 \mu \mathrm{g} / \mathrm{mL}$ of actinomycin D (Sigma) was added to cells and RNA extracted at the time points indicated using Tri Reagent (Sigma). Levels of RNAs of interest were determined by qRT-PCR using SuperScript III Platinum SYBR Green One-Step qRT-PCR Kit (Thermo-Fisher). A total of 200 ng of RNA were loaded per well for RNAs of interest and these levels were normalized to $18 \mathrm{~S}$ Ribosomal 
RNA, for which 1 ng of total RNA was loaded per well (1 in 200 dilution).

\section{Recombinant protein purification}

The full-length human TRIM25 and RBD mutant were cloned into pET30a, which was transformed into BL21 gold cells. Bacteria was grown in Superbroth in the presence of kanamycin at $37^{\circ} \mathrm{C}$ until the $\mathrm{OD}_{600}$ reached 0.6. The bacteria were induced by $0.5 \mathrm{mM}$ IPTG and incubated for 24 hours at $30{ }^{\circ} \mathrm{C}$ with agitation. Pellets were lysed with a cell disruptor in lysis buffer $(20 \mathrm{mM} \mathrm{NaPPi}$ (pH 7.4), $500 \mathrm{mM} \mathrm{NaCl}, 5 \mathrm{mM} \mathrm{MgCl}$, protease inhibitor, DNase, and RNase or benzonase), and lysates were loaded onto an IMAC HiTrap 1-mL FF column in binding buffer (20 mM NaPPi (pH 7.4), $500 \mathrm{mM} \mathrm{NaCl}$, $10 \mathrm{mM}$ imidazole) and eluted with increasing amounts of elution buffer (20 mM NaPPi (pH 7.4), $500 \mathrm{mM} \mathrm{NaCl}$, $500 \mathrm{mM}$ imidazole). Protein fractions were further purified with a gel exclusion column (Superdex 200 30/100 GL or HiLoad Superdex 200 16/60) in gel exclusion buffer (10 mM Tris (pH 8.0), $300 \mathrm{mM} \mathrm{NaCl,} 2 \mathrm{mM}$ DTT). Selected fractions were pooled and concentrated using Vivaspin 5 30,000 MWCO (Sartorius Stedim UK Ltd.). His-Lin28s was produced and purified as previously specified [53].

\section{Thermal denaturation assay}

Thermal denaturation assays were performed on a BioRad IQ5 ICycler. Recombinant proteins $(\sim 4 \mu \mathrm{M})$ were mixed with Sypro orange $(\times 5)$ in a $50-\mu \mathrm{L}$ reaction. Fluorescent readings were taken every 30 seconds between $20{ }^{\circ} \mathrm{C}$ and $70{ }^{\circ} \mathrm{C}$. The melting temperature was determined from the maximum of the first derivative of the melting curve, and the mean melting temperature of three replicates was calculated for each protein.

\section{Size exclusion chromatography with multi-angle light scattering}

Mass measurements were performed on a Viscotek MALS-20 and VE3580 RI detector attached to an ÄKTA 10 Purifier with microflow components. Protein samples were run at approximately $0.75 \mathrm{mg} / \mathrm{mL}$ on a Superdex200 Increase 10/300 GL column, running at $1.0 \mathrm{~mL} /$ min in gel exclusion buffer (with $0.5 \mathrm{mM}$ TCEP instead of DTT) with $100-\mu \mathrm{L}$ injections.

\section{TRIM25 CRISPR/Cas9 knockout}

HeLa cells were transfected with $200 \mathrm{ng}$ of GeneArt CRISPR Nuclease mRNA (Thermo-Fisher) in addition to $50 \mathrm{ng}$ of each of two sgRNAs (TRIM25 Left sgRNA CCACGTTGCACAGCACCGTGTTC and TRIM25 Right sgRNA CTGCGGTCGCGCCTGGTAGACGG) targeting sequences in exon 1 of TRIM25. After 24 hours, cells were seeded to a 96 -well plate such that there was on average 1 cell per well, and the cells were grown until colonies were visible to the naked eye. Next, cells were split into two 96well plates, one of which was used for a dot blot analysis. For the dot blot analysis, cells were washed twice in PBS prior to the addition of $30 \mu \mathrm{L}$ of Passive Lysis Buffer (Promega) per well and incubation for 15 minutes at room temperature. Two microliters of protein from each well was spotted directly onto a nitrocellulose membrane, followed by western blotting, as described above. Selected clones were seeded from the second 96-well plate into 6well plates, grown, and TRIM25 levels were validated by standard western blotting.

\section{CLIP oligonucleotides}

L5Ba: 5' -invddT-ACACrGrArCrGrCrUrCrUrUrCrCrGrA rUrCrUrNrNrNrArGrArGrCrN-OH-3'. L5Bb: 5' -invddTACACrGrArCrGrCrUrCrUrUrCrCrGrArUrCrUrNrNrNr GrUrGrArGrCrN-OH-3'. L5Bc: 5' -invddT-ACACrGrArC rGrCrUrCrUrUrCrCrGrArUrCrUrNrNrNrCrArCrUrArG rCrN-OH-3' . L5Bd: 5'-invddT-ACACrGrArCrGrCrUr CrUrUrCrCrGrArUrCrUrNrNrNrUrCrUrCrUrArGrCrNOH-3'. 3' adapter: 5'App-NAGATCGGAAGAGCACAC GTCTG-ddC 3'. RT Oligo: 5' -CAGACGTGTGCTCTTC CGATCT-3'.

\section{PCR oligonucleotides}

P5_Forward: 5'-AATGATACGGCGACCACCGAGATC TACACTCTTTCCCTACACGACGCTCTTCCGATCT-

3'. P3_barcode_Reverse: BC1: 5'CAAGCAGAAGACG GCATACGAGATCGTGATGTGACTGGAGTTCAGAC GTGTGCTCTTCCGATCT-3' (has indexing barcode IDX1). BC2: 5'CAAGCAGAAGACGGCATACGAGATA CATCGGTGACTGGAGTTCAGACGTGTGCTCTTCC GATCT-3' (has indexing barcode IDX2).

\section{Additional file}

Additional file 1: Figure S1. Thermal denaturation assays and size exclusion chromatography with multi-angle light scattering analyses show that $\triangle \mathrm{RBD}$ deletion does not influence the overall shape and multimerization of purified His-TRIM25 $\triangle$ RBD. Figure S2. Generation and characterization of TRIM25 KO HeLa cell line. Figure S3. T7-TRIM25 CLIP-seq and T7-TRIM25 RNA immunoprecipitation results. Figure S4. T7-TRIM25 CLIP-seq experiments are reproducible, based on three independent experiments. Figure S5. No correlation of TRIM25 CLIP-seq cluster intensities with transcript abundance in HeLa cells. Figure S6. Gene Ontology-term annotation of TRIM25-bound transcripts and the ab initio-derived TRIM25-binding binding sequences cluster in three motif clusters. Figure S7. TRIM25 does not influence RNA stability of selected targets. Figure S8. TRIM25 undergoes ubiquitination. (PDF 9873 kb)

Additional file 2: Table S1. Supplementary data showing protein names, SILAC ratios, peptide counts, and peptide intensities identified by SILAC MS in T7-TRIM25, T7-TRIM25 2 RBD, and T7-TRIM25 + RNases co-immunoprecipitation assays. (XLSX $22 \mathrm{~kb}$ )

Additional file 3: Table S2. Supplementary data showing protein names, SILAC ratios (more than two-fold), peptide counts, and peptide intensities identified by SILAC MS in wild-type and TRIM25 KO HeLa cells. (XLSX $33 \mathrm{~kb})$ 
Additional file 4: Table S3. Supplementary data showing gene names and number of RNA reads derived from three independent T7-TRIM25 CLIP-seq experiments. (XLSX $117 \mathrm{~kb}$ )

\section{Acknowledgments}

We thank Hywel Dunn-Davies for providing a script for the analysis of the distribution of Clip-seq clusters in total RNA and in mRNA. We thank Finn Grey and Sam J. Wilson for the ZAP (ZC3HAV1) plasmid [54].

\section{Funding}

GH was a recipient of a Wellcome Trust PhD studentship (105246). JR was supported by a Wellcome Trust Senior Research Fellowship (084229). JSN was a recipient of a Wellcome Trust PhD Studentship (096996). SG was supported by a Wellcome Trust Career Development Fellowship (091549). GM was a recipient of an MRC Career Development Award (G10000564). This work was also supported by two Wellcome Trust Centre Core Grants (077707 and 092076) and by a Wellcome Trust instrument Grant (091020). Next generation sequencing was carried out by Edinburgh Genomics (MiSeq), The University of Edinburgh. Edinburgh Genomics is partly supported through core grants from NERC (R8/H10/56), MRC (MR/K001744/1), and BBSRC (BB/ J004243/1).

\section{Availability of data and materials}

All data generated or analyzed during this study are included in this published article (and its additional files). The mass spectrometry proteomics data have been deposited in the ProteomeXchange Consortium via the PRIDE [55] partner repository with the dataset identifier PXD007960. Requests for material should be made to the corresponding author.

\section{Authors' contributions}

GM conceived of the study. GM, NRC, GH, MT, and PK designed the experiments, NRC, GH, MT, PK, and CS performed experiments. GM, NRC, GH, MT, PK, SW, SG, CS, JR, and AC analyzed the data. NRC and GM wrote the manuscript with input from other authors. All authors read and approved the final manuscript.

\section{Ethics approval and consent to participate}

Not applicable

\section{Competing interests}

The authors declare that they have no competing interests.

\section{Publisher's Note}

Springer Nature remains neutral with regard to jurisdictional claims in published maps and institutional affiliations.

\section{Author details \\ ${ }^{1}$ Wellcome Centre for Cell Biology, University of Edinburgh, Michael Swann Building, Edinburgh EH9 3BF, UK. ${ }^{2}$ Institute of Quantitative Biology, Biochemistry and Biotechnology, University of Edinburgh, Roger Land Building, Edinburgh EH9 3FF, UK. ${ }^{3}$ Centre for Synthetic and Systems Biology (SynthSys), University of Edinburgh, CH Waddington Building, Edinburgh EH9 3BF, UK. ${ }^{4}$ Department of Biotechnology, Technische Universität Berlin, 13353 Berlin, Germany. ${ }^{5}$ Department of Biochemistry, University of Oxford, South Parks Road, Oxford OX1 3QU, UK.}

Received: 18 October 2017 Accepted: 19 October 2017 Published online: 08 November 2017

\section{References}

1. Castello A, Fischer B, Eichelbaum K, Horos R, Beckmann BM, Strein C, Davey NE, Humphreys DT, Preiss T, Steinmetz LM, et al. Insights into RNA biology from an atlas of mammalian mRNA-binding proteins. Cell. 2012;149:1393-406.

2. Baltz AG, Munschauer M, Schwanhausser B, Vasile A, Murakawa $Y$, Schueler M, Youngs N, Penfold-Brown D, Drew K, Milek M, et al. The mRNA-bound proteome and its global occupancy profile on protein-coding transcripts. Mol Cell. 2012;46:674-90.

3. Kwon SC, Yi H, Eichelbaum K, Fohr S, Fischer B, You KT, Castello A, Krijgsveld J, Hentze MW, Kim VN. The RNA-binding protein repertoire of embryonic stem cells. Nat Struct Mol Biol. 2013;20:1122-30.
4. Liao Y, Castello A, Fischer B, Leicht S, Foehr S, Frese CK, Ragan C, Kurscheid $\mathrm{S}$, Pagler $\mathrm{E}$, Yang $\mathrm{H}$, et al. The cardiomyocyte RNA-binding proteome: links to intermediary metabolism and heart disease. Cell Rep. 2016;16:1456-69.

5. Versteeg GA, Rajsbaum R, Sanchez-Aparicio MT, Maestre AM, Valdiviezo J, Shi M, Inn KS, Fernandez-Sesma A, Jung J, Garcia-Sastre A. The E3-ligase TRIM family of proteins regulates signaling pathways triggered by innate immune pattern-recognition receptors. Immunity. 2013;38:384-98.

6. Rajsbaum R, Garcia-Sastre A, Versteeg GA. TRIMmunity: the roles of the TRIM E3-ubiquitin ligase family in innate antiviral immunity. J Mol Biol. 2014;426: 1265-84.

7. Jefferies C, Wynne C, Higgs R. Antiviral TRIMs: friend or foe in autoimmune and autoinflammatory disease? Nat Rev Immunol. 2011;11:617-25.

8. Ozato K, Shin DM, Chang TH, Morse 3rd HC. TRIM family proteins and their emerging roles in innate immunity. Nat Rev Immunol. 2008;8:849-60.

9. Yoneyama M, Kikuchi M, Natsukawa T, Shinobu N, Imaizumi T, Miyagishi M, Taira K, Akira S, Fujita T. The RNA helicase RIG-I has an essential function in double-stranded RNA-induced innate antiviral responses. Nat Immunol. 2004;5:730-7.

10. Loo YM, Gale Jr M. Immune signaling by RIG-I-like receptors. Immunity. 2011;34:680-92.

11. Gack MU, Shin $Y C$, Joo CH, Urano T, Liang C, Sun L, Takeuchi O, Akira S, Chen Z, Inoue S, Jung JU. TRIM25 RING-finger E3 ubiquitin ligase is essential for RIG-I-mediated antiviral activity. Nature. 2007;446:916-20.

12. Zheng X, Wang X, Tu F, Wang Q, Fan Z, Gao G. TRIM25 is required for the antiviral activity of zinc finger antiviral protein. J Virol. 2017;91(9).

13. Li MM, Lau Z, Cheung P, Aguilar EG, Schneider WM, Bozzacco L, Molina H, Buehler E, Takaoka A, Rice CM, et al. TRIM25 enhances the antiviral action of zinc-finger antiviral protein (ZAP). PLoS Pathog. 2017;13:e1006145.

14. Choudhury NR, Nowak JS, Zuo J, Rappsilber J, Spoel SH, Michlewski G. Trim25 is an RNA-specific activator of Lin28a/TuT4-mediated uridylation. Cell Rep. 2014;9:1265-72.

15. Streich Jr FC, Ronchi VP, Connick JP, Haas AL. Tripartite motif ligases catalyze polyubiquitin chain formation through a cooperative allosteric mechanism. J Biol Chem. 2013;288:8209-21.

16. Koliopoulos MG, Esposito D, Christodoulou E, Taylor IA, Rittinger K. Functional role of TRIM E3 ligase oligomerization and regulation of catalytic activity. EMBO J. 2016;35:1204-18.

17. Li Y, Wu H, Wu W, Zhuo W, Liu W, Zhang Y, Cheng M, Chen YG, Gao N, Yu $\mathrm{H}$, et al. Structural insights into the TRIM family of ubiquitin E3 ligases. Cell Res. 2014;24:762-5.

18. Sanchez JG, Okreglicka K, Chandrasekaran V, Welker JM, Sundquist WI, Pornillos O. The tripartite motif coiled-coil is an elongated antiparallel hairpin dimer. Proc Natl Acad Sci U S A. 2014;111:2494-9.

19. Castello A, Fischer B, Frese CK, Horos R, Alleaume AM, Foehr S, Curk T, Krijgsveld J, Hentze MW. Comprehensive identification of RNA-binding domains in human cells. Mol Cell. 2016;63:696-710.

20. Feracci M, Foot JN, Grellscheid SN, Danilenko M, Stehle R, Gonchar O, Kang HS, Dalgliesh C, Meyer NH, Liu Y, et al. Structural basis of RNA recognition and dimerization by the STAR proteins T-STAR and Sam68. Nat Commun. 2016;7:10355

21. Lunde BM, Moore C, Varani G. RNA-binding proteins: modular design for efficient function. Nat Rev Mol Cell Biol. 2007;8:479-90.

22. da Huang W, Sherman BT, Lempicki RA. Systematic and integrative analysis of large gene lists using DAVID bioinformatics resources. Nat Protoc. 2009;4:44-57.

23. Merico D, Isserlin R, Stueker O, Emili A, Bader GD. Enrichment map: a network-based method for gene-set enrichment visualization and interpretation. PLoS One. 2010;5:e13984.

24. Brannan KW, Jin W, Huelga SC, Banks CA, Gilmore JM, Florens L, Washburn MP, Van Nostrand EL, Pratt GA, Schwinn MK, et al. SONAR discovers RNAbinding proteins from analysis of large-scale protein-protein interactomes. Mol Cell. 2016;64(2):282-93.

25. Webb S, Hector RD, Kudla G, Granneman S. PAR-CLIP data indicate that Nrd1-Nab3-dependent transcription termination regulates expression of hundreds of protein coding genes in yeast. Genome Biol. 2014;15:R8.

26. Walsh LA, Alvarez MJ, Sabio EY, Reyngold M, Makarov V, Mukherjee S, Lee KW, Desrichard A, Turcan S, Dalin MG, et al. An integrated systems biology approach identifies TRIM25 as a key determinant of breast cancer metastasis. Cell Rep. 2017;20:1623-40.

27. Inn KS, Gack MU, Tokunaga F, Shi M, Wong LY, Iwai K, Jung JU. Linear ubiquitin assembly complex negatively regulates RIG-I- and TRIM25mediated type I interferon induction. Mol Cell. 2011;41:354-65. 
28. Takata MA, Goncalves-Carneiro D, Zang TM, Soll SJ, York A, Blanco-Melo D, Bieniasz PD. CG dinucleotide suppression enables antiviral defence targeting non-self RNA. Nature. 2017;550:124-7.

29. Ferber $\mathrm{S}$, Ciechanover A. Transfer RNA is required for conjugation of ubiquitin to selective substrates of the ubiquitin- and ATP-dependent proteolytic system. J Biol Chem. 1986:261:3128-34.

30. Mayr C, Bartel DP. Widespread shortening of $3^{\prime} U$ TRs by alternative cleavage and polyadenylation activates oncogenes in cancer cells. Cell. 2009;138:673-84.

31. Gruber AR, Martin G, Muller P, Schmidt A, Gruber AJ, Gumienny R, Mittal N, Jayachandran R, Pieters J, Keller W, et al. Global 3' UTR shortening has a limited effect on protein abundance in proliferating $T$ cells. Nat Commun. 2014:5:5465

32. Loedige I, Gaidatzis D, Sack R, Meister G, Filipowicz W. The mammalian TRIM-NHL protein TRIM71/LIN-41 is a repressor of mRNA function. Nucleic Acids Res. 2013:41:518-32.

33. Yoon JH, Abdelmohsen K, Kim J, Yang X, Martindale JL, Tominaga-Yamanaka K, White EJ, Orjalo AV, Rinn JL, Kreft SG, et al. Scaffold function of long noncoding RNA HOTAIR in protein ubiquitination. Nat Commun. 2013;4:2939.

34. Leppek K, Schott J, Reitter S, Poetz F, Hammond MC, Stoecklin G. Roquin promotes constitutive mRNA decay via a conserved class of stem-loop recognition motifs. Cell. 2013;153:869-81.

35. Kawai S, Amano A. BRCA1 regulates microRNA biogenesis via the DROSHA microprocessor complex. J Cell Biol. 2012;197:201-8.

36. Heikel G, Choudhury NR, Michlewski G. The role of Trim25 in development, disease and RNA metabolism. Biochem Soc Trans. 2016:44:1045-50.

37. Inoue S, Orimo A, Hosoi T, Kondo S, Toyoshima H, Kondo T, Ikegami A Ouchi Y, Orimo H, Muramatsu M. Genomic binding-site cloning reveals an estrogen-responsive gene that encodes a RING finger protein. Proc Natl Acad Sci U S A. 1993;90:11117-21.

38. Orimo A, Inoue S, Ikeda K, Noji S, Muramatsu M. Molecular cloning, structure, and expression of mouse estrogen-responsive finger protein Efp. Co-localization with estrogen receptor mRNA in target organs. J Biol Chem. 1995;270:24406-13.

39. Orimo A, Inoue S, Minowa O, Tominaga N, Tomioka Y, Sato M, Kuno J, Hiroi $H$, Shimizu Y, Suzuki M, et al. Underdeveloped uterus and reduced estrogen responsiveness in mice with disruption of the estrogen-responsive finger protein gene, which is a direct target of estrogen receptor alpha. Proc Natl Acad Sci U S A. 1999;96:12027-32.

40. Ikeda K, Orimo A, Higashi Y, Muramatsu M, Inoue S. Efp as a primary estrogen-responsive gene in human breast cancer. FEBS Lett. 2000;472:9-13.

41. Urano T, Saito T, Tsukui T, Fujita M, Hosoi T, Muramatsu M, Ouchi Y, Inoue S. Efp targets 14-3-3 sigma for proteolysis and promotes breast tumour growth. Nature. 2002;417:871-5.

42. Thomson SD, Ali S, Pickles L, Taylor J, Pace PE, Lymboura M, Shousha S, Coombes RC. Analysis of estrogen-responsive finger protein expression in benign and malignant human breast. Int J Cancer. 2001;91:152-8.

43. Suzuki T, Urano T, Tsukui T, Horie-Inoue K, Moriya T, Ishida T, Muramatsu M, Ouchi $Y$, Sasano $H$, Inoue $S$. Estrogen-responsive finger protein as a new potential biomarker for breast cancer. Clin Cancer Res. 2005;11:6148-54.

44. Zeng W, Sun L, Jiang X, Chen X, Hou F, Adhikari A, Xu M, Chen ZJ. Reconstitution of the RIG-I pathway reveals a signaling role of unanchored polyubiquitin chains in innate immunity. Cell. 2010;141:315-30.

45. Gack MU, Albrecht RA, Urano T, Inn KS, Huang IC, Carnero E, Farzan M Inoue S, Jung JU, Garcia-Sastre A. Influenza A virus NS1 targets the ubiquitin ligase TRIM25 to evade recognition by the host viral RNA sensor RIG-I. Cell Host Microbe. 2009;5:439-49.

46. Donelan NR, Basler CF, Garcia-Sastre A. A recombinant influenza A virus expressing an RNA-binding-defective NS1 protein induces high levels of beta interferon and is attenuated in mice. J Virol. 2003;77:13257-66.

47. Hu Y, Li W, Gao T, Cui Y, Jin Y, Li P, Ma Q, Liu X, Cao C. SARS coronavirus nucleocapsid inhibits type I interferon production by interfering with TRIM25-mediated RIG-I ubiquitination. J Virol. 2017;91(8).

48. Ghosh P, Sowdhamini R. Genome-wide survey of putative RNA-binding proteins encoded in the human proteome. Mol Biosyst. 2016:12:532-40.

49. Choudhury NR, de Lima AF, de Andres-Aguayo L, Graf T, Caceres JF, Rappsilber J, Michlewski G. Tissue-specific control of brain-enriched miR-7 biogenesis. Genes Dev. 2013;27:24-38.

50. Michlewski G, Caceres JF. RNase-assisted RNA chromatography. RNA. 2010; 16:1673-8.

51. Moore MJ, Zhang C, Gantman EC, Mele A, Darnell JC, Darnell RB. Mapping Argonaute and conventional RNA-binding protein interactions with RNA at single-nucleotide resolution using HITS-CLIP and CIMS analysis. Nat Protoc. 2014;9:263-93.

52. Kilchert C, Wittmann S, Passoni M, Shah S, Granneman S, Vasiljeva L. Regulation of mRNA levels by decay-promoting introns that recruit the exosome specificity factor Mmi1. Cell Rep. 2015;13:2504-15.

53. Nowak JS, Hobor F. Downie Ruiz Velasco A, Choudhury NR, Heikel G, Kerr A, Ramos A, Michlewski G. Lin28a uses distinct mechanisms of binding to RNA and affects positively and negatively miRNA levels. RNA. 2017;23(3):317-32.

54. Schoggins JW, Wilson SJ, Panis M, Murphy MY, Jones CT, Bieniasz P, Rice $C M$. A diverse range of gene products are effectors of the type I interferon antiviral response. Nature. 2011:472:481-5.

55. Vizcaino JA, Csordas A, del-Toro N, Dianes JA, Griss J, Lavidas I, Mayer G, Perez-Riverol Y, Reisinger F, Ternent T, et al. 2016 update of the PRIDE database and its related tools. Nucleic Acids Res. 2016;44:D447-456.

\section{Submit your next manuscript to BioMed Central and we will help you at every step:}

- We accept pre-submission inquiries

- Our selector tool helps you to find the most relevant journal

- We provide round the clock customer support

- Convenient online submission

- Thorough peer review

- Inclusion in PubMed and all major indexing services

- Maximum visibility for your research

Submit your manuscript at www.biomedcentral.com/submit
Biomed Central 